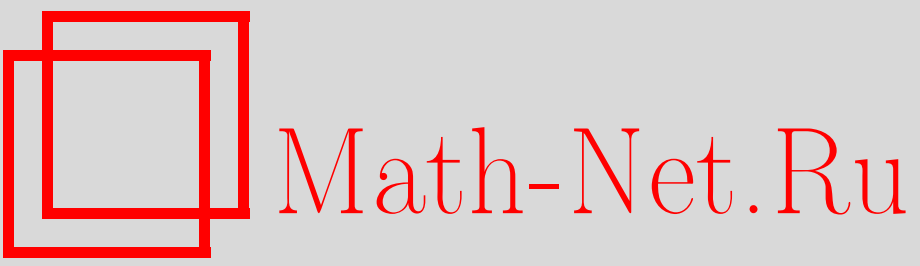

Г. В. Сандраков, Осреднение вариационных неравенств для задач нелинейной диффузии в перфорированных областях, Изв. РАН. Сер. матем., 2005, том 69, выпуск 5, 179-204

DOI: https://doi.org/10.4213/im660

Использование Общероссийского математического портала Math-Net.Ru подразумевает, что вы прочитали и согласны с пользовательским соглашением

http: //www . mathnet.ru/rus/agreement

Параметры загрузки:

IP : 3.91 .87 .62

26 апреля 2023 г., 12:27:09 


\title{
Г.В. Сандраков
}

\section{Осреднение вариационных неравенств для задач нелинейной диффузии в перфорированных областях}

\begin{abstract}
Рассмотрено осреднение задач нелинейной диффузии в периодически перфорированных областях с различными граничными условиями. Такие задачи формулируются в виде вариационных неравенств, определяемых нелинейным строго монотонным оператором второго порядка с периодическими быстроосциллирующими коэффициентами. Доказаны утверждения о соответствующей сходимости решений этих задач к решениям двухмасштабных и макромасштабных предельных вариационных неравенств. Представлены методы вывода таких предельных вариационных неравенств. Для потенциальных операторов доказаны утверждения о связи полученных предельных вариационных неравенств с двухмасштабными и макромасштабными задачами минимизации.

Библиография: 28 наименований.
\end{abstract}

\section{§1. Введение}

В настоящей работе рассматриваются вопросы осреднения вариационных неравенств для задач с условием Синьорини на внутренней гранище периодически перфорированных областей, моделируюших пористые среды, и однородными условиями Дирихле на внешней границе. Рассмотрены также вариационные неравенства для задач с препятствием в перфорированных областях. Такие вариационные неравенства являются естественным обобщением задач минимизации с односторонними ограничениями на решения (см., например, [1]).

Пусть $n \geqslant 2$ - целое число. Периодически перфорированную область $\Omega_{\varepsilon}$, зависящую от малого положительного параметра $\varepsilon$, принято определять как пересечение ограниченной области $\Omega$ (с липшицевой границей $\partial \Omega$ ) из $\mathbb{R}^{n}$ и открытого множества $F_{\varepsilon}$, имеющего период $\varepsilon$. Таким образом, если $F_{1}$ является открытым 1-периодическим подмножеством $\mathbb{R}^{n}$, то

$$
\Omega_{\varepsilon}=\Omega \cap F_{\varepsilon},
$$

где $F_{\varepsilon} \equiv \varepsilon F_{1}=\left\{\varepsilon x: x=\left(x_{1}, \ldots, x_{n}\right) \in F_{1}\right\}$. Здесь и далее 1-периодичность множества (функции) означает периодичность характеристической функции этого множества (этой функции) с периодом 1 по каждому $x_{i}, i=1, \ldots, n$. Таким образом, $Y \equiv[0,1]^{n}$ является ячейкой периодичности, и множество $F_{1}$ вполне определяется множеством $F \equiv Y \cap F_{1}$.

Предполагается, что множества $F$ и $Y \backslash F$ имеют положительные меры Лебега в $\mathbb{R}^{n}$ и что множество $F_{1}$ является связным. Кроме того, отождествляя противоположные грани ячейки периодичности $Y$ и используя 1-периодичность, множество $F$ можно рассматривать как подмножество $n$-мерного тора. Предполагается, что такое подмножество $F$ является подмногообразием тора с липшицевой границей $S \equiv \partial F$. При этом из связности $F_{1}$ следует связность $F$, а из определения 
многообразия с границей (как множества, допускающего конечное число систем локальных координат, удовлетворяющих предположениям, приведенным, например, в $[2$, гл. $1, \S 1.6])$ следует компактность многообразия $F$ и гранищы $S$.

Граница $\partial \Omega_{\varepsilon}$ перфорированной области $\Omega_{\varepsilon}$ допускает разбиение на "внутреннюю” часть $S_{\varepsilon} \equiv \partial \Omega_{\varepsilon} \backslash \partial \Omega$ и “внешнюю" часть $\partial \Omega_{\varepsilon} \cap \partial \Omega$. Следуя, например, в работе [3], определим для области $\Omega_{\varepsilon}$ пространство (функций, удовлетворяющих однородным условиям Дирихле на внешней границе)

$$
H_{D}^{1}\left(\Omega_{\varepsilon}\right) \equiv\left\{v \in H^{1}\left(\Omega_{\varepsilon}\right): v=0 \text { на } \partial \Omega_{\varepsilon} \cap \partial \Omega\right\},
$$

которому будут принадлежать решения рассматриваемых вариационных неравенств. Отметим, что для фиксированного $\varepsilon$ множество $\partial \Omega_{\varepsilon} \cap \partial \Omega$ является замкнутым, и поэтому для $v \in H^{1}\left(\Omega_{\varepsilon}\right)$ определено понятие однородных условий Дирихле на каждой компоненте этого множества (см., например, [4]).

Рассмотрим $v \in H_{D}^{1}\left(\Omega_{\varepsilon}\right)$ и обозначим через $\tilde{v}$ продолжение этой функции нулем на $F_{\varepsilon} \backslash \Omega_{\varepsilon}$. Как отмечено в [3] (доказательство леммы 3.3), продолжение $\tilde{v}$ принадлежит $H^{1}\left(F_{\varepsilon}\right)$. Последнее утверждение из [3] имеет некоторые полезные следствия, используемые далее.

Фиксируем $\varepsilon$. Учитывая ограниченность области $\Omega$, можно считать, что найдется такое положительное $b=\varepsilon z$ с целочисленным $z$, для которого $\Omega \subset[0, b]^{n}$. Отождествляя противоположные грани куба $[0, b]^{n}$ и используя $\varepsilon$-периодичность множества $F_{\varepsilon}$, можно рассматривать $F_{\varepsilon}^{b}=F_{\varepsilon} \cap[0, b]^{n}$ как подмногообразие (соответствующего) тора с липшицевой гранищей $S_{\varepsilon}^{b}=\partial F_{\varepsilon}^{b}$ в силу предположений. Тогда $\tilde{v} \in H^{1}\left(F_{\varepsilon}^{b}\right)$ для $v \in H_{D}^{1}\left(\Omega_{\varepsilon}\right)$, поскольку $\tilde{v}=0$ в $F_{\varepsilon} \backslash \Omega_{\varepsilon}$ и определен след $\left.\tilde{v}\right|_{S_{\varepsilon}^{b}}$ как элемент пространства $H^{1 / 2}\left(S_{\varepsilon}^{b}\right)$ (см., например, [5], [6]). Также известно [6], что оператор вычисления такого следа является непрерывным оператором из $H^{1}\left(F_{\varepsilon}^{b}\right)$ в $H^{1 / 2}\left(S_{\varepsilon}^{b}\right)$. Точнее, для липшицевых областей последнее утверждение доказано, например, в [7], а для подмногообразий с липшицевыми границами это утверждение проверяется с помощью подходящей локализации (см., например, в [5]).

Следовательно, для $v \in H_{D}^{1}\left(\Omega_{\varepsilon}\right)$ определен след $\left.v\right|_{S_{\varepsilon}}$ как элемент пространства $H^{1 / 2}\left(S_{\varepsilon}\right)$, поскольку $\left.\tilde{v}\right|_{S_{\varepsilon}^{b} \backslash S_{\varepsilon}}=0$ в силу определений, а непрерывность соответствующего оператора (вычисления) следа подразумевает замкнутость в $H_{D}^{1}\left(\Omega_{\varepsilon}\right)$ множества

$$
K \equiv\left\{v \in H_{D}^{1}\left(\Omega_{\varepsilon}\right): v \geqslant 0 \text { п.в. на } S_{\varepsilon}\right\} .
$$

Отметим, что в силу (локально конечной) липшицевости множества $F_{\varepsilon}$ для фиксированного $\varepsilon$ на $S_{\varepsilon}$ (точнее, на $S_{\varepsilon}^{b}$ ) определена естественная мера, и поэтому определено понятие п.в. (почти всюду относительно этой меры) на $S_{\varepsilon}$, например, в соответствии с $[2$, гл. $1, \S 1.6]$. Фактически из лишшицевости множества $F_{\varepsilon}$ следует также неравенство Пуанкаре:

$$
\|v\|_{L^{2}\left(\Omega_{\varepsilon}\right)} \leqslant C\|\nabla v\|_{L^{2}\left(\Omega_{\varepsilon}\right)^{n}} \text { для } v \in H_{D}^{1}\left(\Omega_{\varepsilon}\right) .
$$

В работе [3] доказано, что постоянную $C$ в этом неравенстве можно выбрать не зависяшей от положительного параметра $\varepsilon$ (при $0<\varepsilon \leqslant \varepsilon_{0}$ для некоторого положительного $\left.\varepsilon_{0}\right)$.

Пусть заданы функция $f \in L^{2}(\Omega)$ и последовательность функций $\left\{\psi_{\varepsilon}\right\} \subset$ $H_{D}^{1}\left(\Omega_{\varepsilon}\right)$, зависящая от малого положительного параметра $\varepsilon$ и равномерно ограниченная относительно этого параметра $\left(\left\|\psi_{\varepsilon}\right\|_{H^{1}\left(\Omega_{\varepsilon}\right)} \leqslant C\right.$, где постоянная $C$ не 
зависит от $\varepsilon$ ). Рассмотрим следуюшую задачу, зависяшую от $\varepsilon$ и формулируемую в виде вариационного неравенства для $\hat{u}_{\varepsilon} \in K_{\varepsilon}$ :

$$
\begin{gathered}
\int_{\Omega_{\varepsilon}} a\left(\frac{x}{\varepsilon}, \nabla \hat{u}_{\varepsilon}\right)\left(\nabla \hat{v}-\nabla \hat{u}_{\varepsilon}\right) d x \geqslant \int_{\Omega_{\varepsilon}} f\left(\hat{v}-\hat{u}_{\varepsilon}\right) d x, \\
\forall \hat{v} \in K_{\varepsilon} \equiv\left\{\hat{v} \in H_{D}^{1}\left(\Omega_{\varepsilon}\right): \hat{v} \geqslant \psi_{\varepsilon} \text { п.в. на } S_{\varepsilon}\right\},
\end{gathered}
$$

где вектор-функция $a(y, \xi)$ определена на $F_{1}$ и является 1 -периодической по $y \in F_{1}$ для каждого $\xi \in \mathbb{R}^{n}$. Следовательно, $a(y, \xi)$ для каждого $\xi \in \mathbb{R}^{n}$ вполне определяется своим ограничением на $F$. Предполагается, что $a(y, \xi)$ для каждого $\xi \in \mathbb{R}^{n}$ является измеримой на $F, a(y, 0)=0$ и

$$
\begin{gathered}
0<(a(y, \xi)-a(y, \zeta))(\xi-\zeta), \quad \xi \neq \zeta, \\
\alpha|\xi|^{2} \leqslant a(y, \xi) \xi, \quad|a(y, \xi)-a(y, \zeta)| \leqslant \beta|\xi-\zeta| \\
\text { п.в. в } F \forall \xi, \zeta \in \mathbb{R}^{n},
\end{gathered}
$$

где $\alpha$ и $\beta$ - заданные положительные постоянные.

Таким образом, для фиксированного $\varepsilon$ вектор-функция $a(y, \xi)$ определяет строго монотонный, непрерывный и коэрцитивный оператор в неравенстве (1.5) (см., например, [1, гл. 2, §8]). Известно [1], что сушествует единственное (для фиксированного $\varepsilon$ ) решение вариационного неравенства (1.5) и посредством замены $u_{\varepsilon}=\hat{u}_{\varepsilon}-\psi_{\varepsilon}, \quad v=\hat{v}-\psi_{\varepsilon}$ это неравенство эквивалентно вариационному неравенству для $u_{\varepsilon} \in K$ :

$$
\int_{\Omega_{\varepsilon}} a\left(\frac{x}{\varepsilon}, \nabla \psi_{\varepsilon}+\nabla u_{\varepsilon}\right)\left(\nabla v-\nabla u_{\varepsilon}\right) d x \geqslant \int_{\Omega_{\varepsilon}} f\left(v-u_{\varepsilon}\right) d x \quad \forall v \in K,
$$

где множество $K$ определено в (1.3) и является замкнутым (в силу свойств оператора следа на $S_{\varepsilon}$ ) выпуклым конусом с вершиной в нуле.

Также известно [1], что вариационное неравенство (1.7) эквивалентно следующему вариационному неравенству $u_{\varepsilon} \in K$ :

$$
\int_{\Omega_{\varepsilon}} a\left(\frac{x}{\varepsilon}, \nabla \psi_{\varepsilon}+\nabla v\right)\left(\nabla v-\nabla u_{\varepsilon}\right) d x \geqslant \int_{\Omega_{\varepsilon}} f\left(v-u_{\varepsilon}\right) d x \quad \forall v \in K .
$$

Эти эквивалентности означают (для фиксированного $\varepsilon$ ), что $u_{\varepsilon} \in K$ является решением задачи (1.7), если и только если $u_{\varepsilon} \in K$ является решением задачи (1.8) и если и только если $\hat{u}_{\varepsilon}=u_{\varepsilon}+\psi_{\varepsilon} \in K_{\varepsilon}$ является решением задачи (1.5).

В соответствии с [1] решение $u_{\varepsilon} \in K_{\varepsilon}$ вариационного неравенства (1.5) характеризуется также следуюшими условиями (возможно, при дополнительных условиях регулярности на исходные данные):

$$
\begin{gathered}
-\operatorname{div}\left(a\left(\frac{x}{\varepsilon}, \nabla \hat{u}_{\varepsilon}\right)\right)=f \text { в } \Omega_{\varepsilon}, \\
\hat{u}_{\varepsilon}=0 \text { на } \partial \Omega_{\varepsilon} \cap \partial \Omega, \\
\left(\hat{u}_{\varepsilon}-\psi_{\varepsilon}\right)\left(\nu_{\varepsilon} \cdot a\left(\frac{x}{\varepsilon}, \nabla \hat{u}_{\varepsilon}\right)\right)=0 \text { на } S_{\varepsilon}, \\
\hat{u}_{\varepsilon} \geqslant \psi_{\varepsilon} \text { и }\left(\nu_{\varepsilon} \cdot a\left(\frac{x}{\varepsilon}, \nabla \hat{u}_{\varepsilon}\right)\right) \geqslant 0 \text { на } S_{\varepsilon},
\end{gathered}
$$

где $\nu_{\varepsilon}$. обозначает скалярное умножение на вектор внешней нормали к гранище $S_{\varepsilon}$. 
Задачу (1.9) принято называть задачей с односторонними ограничениями на границе $S_{\varepsilon}$ или задачей с условиями Синьорини на $S_{\varepsilon}$. В соответствии с условиями этой задачи гранища $S_{\varepsilon}$ допускает разбиение на две части:

$$
S_{\varepsilon}^{0}=\left\{\left.\left(\hat{u}_{\varepsilon}-\psi_{\varepsilon}\right)\right|_{S_{\varepsilon}}=0\right\} \text { и } S_{\varepsilon}^{+}=\left\{\left.\left(\hat{u}_{\varepsilon}-\psi_{\varepsilon}\right)\right|_{S_{\varepsilon}}>0\right\}
$$

и это разбиение является одним из неизвестных задачи (см., например, [1]). Таким образом, задача (1.9) может быть интерпретирована как модель для установившейся нелинейной диффузии (или теплопроводности) в пористой среде, на одной части границы которой заданы условия Дирихле $\left(\hat{u}_{\varepsilon}=\psi_{\varepsilon}\right)$, а на другой части условия Неймана

$$
\left(\nu_{\varepsilon} \cdot a\left(\frac{x}{\varepsilon}, \nabla \hat{u}_{\varepsilon}\right)\right)=0 \text { на } S_{\varepsilon}^{+} \text {. }
$$

Соответственно, вариационное неравенство (1.5) является “обобщенной” постановкой задачи (1.9).

Основные результаты работы составляют утверждения о предельном поведении решений вариационного неравенства (1.5) (и, соответственно, решений эквивалентных вариационных задач (1.7) и (1.8)). Будет получено осредненное (предельное при $\varepsilon \rightarrow 0$ ) вариационное неравенство и доказана теорема о соответствуюшей сходимости последовательности решений к решению осредненной вариационной задачи. В формулировке этой теоремы используется понятие двухмасштабной сходимости (подробности о такой сходимости можно найти в [8], [9]).

Последовательность $\left\{v_{\varepsilon}(x)\right\} \subset L^{2}(\Omega)$ называется двухмасштабно сходящейся $\kappa v_{0}(x, y) \in L^{2}(\Omega \times Y)$ (обозначение $\left.v_{\varepsilon} \stackrel{2}{\longrightarrow} v_{0}\right)$ при $\varepsilon \rightarrow 0$, если

$$
\lim _{\varepsilon \rightarrow 0} \int_{\Omega} v_{\varepsilon}(x) \varphi\left(x, \frac{x}{\varepsilon}\right) d x=\int_{\Omega} \int_{Y} v_{0}(x, y) \varphi(x, y) d y d x
$$

для каждого $\varphi(x, y) \in L_{P}^{2}(Y ; C(\bar{\Omega}))$. Здесь и далее индекс $P$ означает 1 -периодичность по $y \in \mathbb{R}^{n}$. Покомпонентно такая сходимость определяется и для вектор-функций. Известно [8], [9], что каждая равномерно ограниченная последовательность $\left\{v_{\varepsilon}\right\} \subset L^{2}(\Omega)$ имеет подпоследовательность, двухмасштабно сходящуюся к некоторому элементу $v_{0}(x, y)$ из $L^{2}(\Omega \times Y)$.

Рассмотрим некоторую функцию $v_{\varepsilon}$, определенную на $\Omega_{\varepsilon}$, например, из $L^{2}\left(\Omega_{\varepsilon}\right)$. Обозначим через $\chi_{\varepsilon} v_{\varepsilon}$ продолжение этой функции нулем на $\Omega \backslash \Omega_{\varepsilon}$. Тогда $\chi_{\varepsilon}=\chi_{\varepsilon} 1$ можно рассматривать как характеристическую функцию множества $\Omega_{\varepsilon}$, и последовательность $\left\{\chi_{\varepsilon} v_{\varepsilon}\right\}$ равномерно ограничена в $L^{2}(\Omega)$, если последовательность $\left\{v_{\varepsilon}\right\}$ равномерно ограничена в $L^{2}\left(\Omega_{\varepsilon}\right)$. Аналогично, для $\varphi \in L_{P}^{2}(F)$ через $\chi \varphi$ обозначим продолжение этой функции нулем на $Y \backslash F$ и через $\chi$ - характеристическую функцию множества $F$.

Из соотношений (1.4), (1.6) и предположений на исходные данные следует, что последовательности $\left\{u_{\varepsilon}\right\}$ и $\left\{\nabla u_{\varepsilon}\right\}$ решений вариационного неравенства (1.7) и градиентов этих решений равномерно ограничены в $L^{2}\left(\Omega_{\varepsilon}\right)$ и $L^{2}\left(\Omega_{\varepsilon}\right)^{n}$ соответственно, и поэтому выполнено следующее утверждение.

УТВЕРЖДЕНИЕ 1.1. Пусть $u_{\varepsilon}(x) \in H_{D}^{1}\left(\Omega_{\varepsilon}\right)$ является решением вариачионного неравенства (1.7). Тогда найдутся такие $\psi_{0}(x), u_{0}(x) \in H_{0}^{1}(\Omega)$ 
и $\psi_{1}(x, y), u_{1}(x, y) \in L^{2}\left(\Omega ; H_{P}^{1}(F) / \mathbb{R}\right)$, что (возможнно, после выделения подпоследовательностей $п р и \varepsilon \rightarrow 0$ )

$$
\begin{array}{cc}
\chi_{\varepsilon} \psi_{\varepsilon} \stackrel{2}{\longrightarrow} \chi \psi_{0}, & \chi_{\varepsilon} u_{\varepsilon} \stackrel{2}{\longrightarrow} \chi u_{0}, \\
\chi_{\varepsilon} \nabla \psi_{\varepsilon} \stackrel{2}{\longrightarrow} \chi \nabla \psi_{0}+\chi \nabla_{y} \psi_{1}, & \chi_{\varepsilon} \nabla u_{\varepsilon} \stackrel{2}{\longrightarrow} \chi \nabla u_{0}+\chi \nabla_{y} u_{1}
\end{array}
$$

$u u_{0} \geqslant 0$ n.в. $в \Omega$.

Доказательства сформулированных здесь утверждений будут приведены в $\S 2$, где имеются и соответствующие ссылки.

Далее предполагается, что $\psi_{0}$ и $\psi_{1}$ из утверждения 1.1 определены однозначно и выполнено равенство

$$
\lim _{\varepsilon \rightarrow 0}\left\|\nabla \psi_{\varepsilon}\right\|_{L^{2}\left(\Omega_{\varepsilon}\right)^{n}}=\left\|\nabla \psi_{0}+\nabla_{y} \psi_{1}\right\|_{L^{2}(\Omega \times F)^{n}}
$$

Эти предположения означают, что последовательность $\left\{\chi_{\varepsilon} \nabla \psi_{\varepsilon}\right\}$ имеет (однозначно определенный) сильный двухмасштабный предел в $L^{2}(\Omega)^{n}($ см. $[9$, теорема 1.8$])$. В частности, равенство (1.11) выполнено при $\psi_{1}=0$, если $\psi_{\varepsilon} \in H_{0}^{1}(\Omega)$ и $\psi_{\varepsilon} \rightarrow \psi_{0}$ сильно в $H_{0}^{1}(\Omega)$ при $\varepsilon \rightarrow 0$.

Будем обозначать через $C_{0}^{\infty}\left(\Omega ; C_{P}^{\infty}(Y) / \chi \mathbb{R}\right)$ множество конечных линейных комбинаций функций вида $\psi_{1}=\psi_{0}^{1}(x) \psi_{P}^{1}(y)$, где $\psi_{0}^{1} \in C_{0}^{\infty}(\Omega)$ и $\psi_{P}^{1} \in C_{P}^{\infty}(Y) / \chi \mathbb{R}$. Здесь по определению $C_{P}^{\infty}(Y) / \chi \mathbb{R}$ рассматривается как множество гладких функций $\varphi \in C_{P}^{\infty}(Y)$, удовлетворяюших равенству $\int_{F} \varphi d y=0$, которое подразумевает, что $\varphi \in H_{P}^{1}(F) / \mathbb{R}$ с точностью до изоморфизма (см., например, [10, гл. 1, замечание 1.4]). Пусть сначала $\psi_{0} \in C_{0}^{\infty}(\Omega)$ и $\psi_{1} \in C_{0}^{\infty}\left(\Omega ; C_{P}^{\infty}(Y) / \chi \mathbb{R}\right)$. Тогда из равенства (1.11) следует (в соответствии с [9]), что

$$
\lim _{\varepsilon \rightarrow 0}\left\|\nabla \psi_{\varepsilon}(x)-\nabla \psi_{0}(x)-\left(\nabla_{y} \psi_{1}\right)\left(x, \frac{x}{\varepsilon}\right)\right\|_{L^{2}\left(\Omega_{\varepsilon}\right)^{n}}=0 .
$$

Введем обозначение $H_{2}=H_{0}^{1}(\Omega) \times L^{2}\left(\Omega ; H_{P}^{1}(F) / \mathbb{R}\right)$. Как отмечено в [9] (доказательство теоремы 2.9), для $\left(v_{0}, v_{1}\right) \in H_{2}$ равенство

$$
\left\|\left(v_{0}, v_{1}\right)\right\|_{2}=\left\|\nabla v_{0}+\nabla_{y} v_{1}\right\|_{L^{2}(\Omega \times F)^{n}}
$$

определяет норму, эквивалентную естественной норме (произведения) на пространстве $\mathrm{H}_{2}$. В соответствии с утверждением 1.1 полезно определить множество

$$
K_{2} \equiv\left\{\left(v_{0}, v_{1}\right) \in H_{2}: v_{0} \geqslant 0 \text { п.в. в } \Omega\right\} \text {, }
$$

содержашее двухмасштабные пределы решений задачи (1.7).

Фиксируем $v_{0} \in C_{0}^{\infty}(\Omega)$ и $v_{1} \in C_{0}^{\infty}\left(\Omega ; C_{P}^{\infty}(Y) / \chi \mathbb{R}\right), v_{0} \geqslant 0$ в $\Omega$. Тогда $\left(v_{0}, v_{1}\right) \in K_{2}$ в силу определений и найдется такая постоянная $\delta$, что $\left|v_{1}\right| \leqslant \delta$ в $\Omega \times Y$, поскольку гладкие функции ограничены на компактных множествах.

Выберем для каждого $\varepsilon$ неотрицательную функцию $e_{\varepsilon} \in W_{0}^{1, \infty}(\Omega)$, равную нулю при $\operatorname{dist}(x, \partial \Omega) \leqslant \varepsilon$ и единище при $\operatorname{dist}(x, \partial \Omega) \geqslant 2 \varepsilon$, где $\operatorname{dist}(x, \partial \Omega)-$ расстояние от точки $x$ до границы $\partial \Omega$. Для достаточно малых $\varepsilon\left(\leqslant \varepsilon_{0}\right.$ для некоторого положительного $\left.\varepsilon_{0}\right)$ такие функции существуют, $\left|\varepsilon \nabla e_{\varepsilon}\right| \leqslant C$ п.в. в $\Omega$ (с постоянной $C$, не зависяшей от $\varepsilon)$, и носитель функции $\nabla e_{\varepsilon}$ содержится 
в приграничной полосе $\Omega_{2 \varepsilon} \subset \Omega$ ширины $2 \varepsilon$ (см., например, [11]). Следовательно, $\lim _{\varepsilon \rightarrow 0}\left\|\varepsilon \nabla e_{\varepsilon}\right\|_{L^{2}(\Omega)^{n}}=0$, и можно считать, что $e_{\varepsilon}=1$ на носителе функции $v_{1}(x, x / \varepsilon)$, поскольку этот носитель компактен в $\Omega$. Тогда $v_{1}(x, x / \varepsilon)+\delta e_{\varepsilon} \geqslant 0$ в $\Omega$, и поэтому выполнены неравенства

$$
\left.\left(v_{1}\left(x, \frac{x}{\varepsilon}\right)+\delta e_{\varepsilon}\right)\right|_{S_{\varepsilon}} \geqslant 0,\left.\quad v_{0}(x)\right|_{S_{\varepsilon}} \geqslant 0
$$

Таким образом, в задаче (1.8) можно выбрать тестовую функцию $v=v_{0}(x)+$ $\varepsilon v_{1}(x, x / \varepsilon)+\varepsilon \delta e_{\varepsilon}$. Учитьвая этот выбор и соотношения (1.6) и (1.12), неравенство из (1.8) можно представить в виде

$$
\begin{gathered}
\int_{\Omega_{\varepsilon}} a\left(\frac{x}{\varepsilon}, \nabla \psi_{0}+\nabla_{y} \psi_{1}^{\varepsilon}+\nabla v_{0}+\nabla_{y} v_{1}^{\varepsilon}\right)\left(\nabla v_{0}+\nabla_{y} v_{1}^{\varepsilon}-\nabla u_{\varepsilon}\right) d x \\
\geqslant \int_{\Omega_{\varepsilon}} f\left(v_{0}-u_{\varepsilon}\right) d x+\sigma_{\varepsilon}
\end{gathered}
$$

где $\lim _{\varepsilon \rightarrow 0} \sigma_{\varepsilon}=0$ и, например, $\nabla_{y} \psi_{1}^{\varepsilon}=\left(\nabla_{y} \psi_{1}\right)(x, x / \varepsilon)$. Следовательно, используя (1.6) и очевидное равенство $\chi_{\varepsilon}=\chi_{\varepsilon}^{2}$, последнее неравенство можно переписать в эквивалентном виде:

$$
\begin{aligned}
\int_{\Omega} \chi_{\varepsilon} a & \left(\frac{x}{\varepsilon}, \nabla \psi_{0}+\nabla_{y} \psi_{1}^{\varepsilon}+\nabla v_{0}+\nabla_{y} v_{1}^{\varepsilon}\right)\left(\chi_{\varepsilon} \nabla v_{0}+\chi_{\varepsilon} \nabla_{y} v_{1}^{\varepsilon}-\chi_{\varepsilon} \nabla u_{\varepsilon}\right) d x \\
& \geqslant \int_{\Omega} f\left(\chi_{\varepsilon} v_{0}-\chi_{\varepsilon} u_{\varepsilon}\right) d x+\sigma_{\varepsilon}
\end{aligned}
$$

Неравенство (1.14) примечательно тем, что в нем можно перейти непосредственно к пределу при $\varepsilon \rightarrow 0$ (возможно, по подпоследовательности из утверждения 1.1), используя только определение (1.10), поскольку $\chi a\left(y, \nabla \psi_{0}+\nabla_{y} \psi_{1}+\right.$ $\left.\nabla v_{0}+\nabla_{y} v_{1}\right) \in L_{P}^{2}\left(Y ; C(\bar{\Omega})^{n}\right)$ в силу (1.6) и известно [9], что $\chi_{\varepsilon} v_{0} \stackrel{2}{\longrightarrow} \chi v_{0}$ и $\chi_{\varepsilon} \nabla v_{0}+$ $\chi_{\varepsilon} \nabla_{y} v_{1}^{\varepsilon} \stackrel{2}{\longrightarrow} \chi \nabla v_{0}+\chi \nabla_{y} v_{1}$.

Таким образом, $u_{0}$ и $u_{1}$, определенные в утверждении 1.1, удовлетворяют неравенству для $\left(u_{0}, u_{1}\right) \in K_{2}$ :

$$
\begin{gathered}
\int_{\Omega} \int_{F} a\left(y, \nabla \psi_{0}+\nabla_{y} \psi_{1}+\nabla v_{0}+\nabla_{y} v_{1}\right)\left(\nabla v_{0}+\nabla_{y} v_{1}-\nabla u_{0}-\nabla_{y} u_{1}\right) d y d x \\
\geqslant \int_{\Omega} \int_{F} f\left(v_{0}-u_{0}\right) d y d x
\end{gathered}
$$

где $\left(v_{0}, v_{1}\right) \in K_{2}$ и учтено, например, равенство $\chi \nabla v_{0}=0$ на $\Omega \times(Y \backslash F)$. Проверяется, что неравенство (1.15) выполнено не только для гладких и финитных $v_{0}$ и $v_{1}$, но и для всех $\left(v_{0}, v_{1}\right) \in K_{2}$. Проверяется также, что неравенство (1.15) выполнено и для произвольных $\psi_{0}$ и $\psi_{1}$, определенных в утверждении 1.1 и удовлетворяюших предположению (1.11). 
УТВЕРЖДЕНИЕ 1.2. Существует единственное решение $\left(u_{0}, u_{1}\right) \in K_{2}$ вариачионного неравенства (1.15) для всех $\left(v_{0}, v_{1}\right) \in K_{2}$, и әто неравенство әквивалентно вариационному неравенству для $\left(u_{0}, u_{1}\right) \in K_{2}$ :

$$
\begin{gathered}
\int_{\Omega} \int_{F} a\left(y, \nabla \psi_{0}+\nabla_{y} \psi_{1}+\nabla u_{0}+\nabla_{y} u_{1}\right)\left(\nabla v_{0}+\nabla_{y} v_{1}-\nabla u_{0}-\nabla_{y} u_{1}\right) d y d x \\
\geqslant \int_{\Omega} \int_{F} f\left(v_{0}-u_{0}\right) d y d x \quad \forall\left(v_{0}, v_{1}\right) \in K_{2} .
\end{gathered}
$$

Таким образом, вариационное неравенство (1.16) определяет предельную двухмасштабную задачу для вариационного неравенства (1.7). Однако цель автора этой работы - получить макромасштабное предельное вариационное неравенство (не зависящее от микромасштабной переменной $y \in F$ ) для задачи (1.7) (и поэтому для эквивалентной задачи (1.5)).

Для этого выберем $v_{0}=u_{0}$ в (1.16) и предположим, что $\xi=\nabla \psi_{0}+\nabla u_{0}$ и $U=\psi_{1}+u_{1}$ (здесь фактически "замораживается" макромасштабная переменная $x \in \Omega$ в (1.16)). Тогда получаем (формально) вариационное неравенство для $U \in H_{P}^{1}(F) / \mathbb{R}:$

$$
\int_{F} a\left(y, \xi+\nabla_{y} U\right)\left(\nabla_{y} V-\nabla_{y} U\right) d y \geqslant 0 \quad \forall V \in H_{P}^{1}(F) / \mathbb{R} .
$$

УТВЕРЖДЕНИЕ 1.3. Для фиксированного $\xi \in \mathbb{R}^{n}$ существует единственное решение $U \in H_{P}^{1}(F) / \mathbb{R}$ вариачионного неравенства $(1.17)$, и это неравенство әквивалентно вариационному уравнению для $U \in H_{P}^{1}(F) / \mathbb{R}$ :

$$
\int_{F} a\left(y, \xi+\nabla_{y} U\right) \nabla_{y} V d y=0 \quad \forall V \in H_{P}^{1}(Y) / \mathbb{R} .
$$

Кроме того, функиия $U=U(\xi)$ является слабо непрерывной как функиия на $\mathbb{R}^{n}$ со значениями в $H_{P}^{1}(F) / \mathbb{R}$.

Для решений задачи (1.18) из (1.6) имеем $\left\|\nabla_{y} U(\xi)\right\|_{L^{2}(F)^{n}}^{2} \leqslant C|\xi|^{2}$ с постоянной $C$, не зависящей от $\xi \in \mathbb{R}^{n}$. Из непрерывности функции $U(\xi)$ и сепарабельности пространства $H_{P}^{1}(F) / \mathbb{R}$ следует сильная измеримость этой функции, и поэтому $U\left(y, \nabla v_{0}(x)\right) \in L^{2}\left(\Omega ; H_{P}^{1}(F) / \mathbb{R}\right)$, если $v_{0} \in H_{0}^{1}(\Omega)$. Таким образом, имеет смысл следующий результат.

УТВЕРЖДЕНИЕ 1.4. Пусть $\left(u_{0}, u_{1}\right) \in K_{2}$ является единственным решением вариационного неравенства (1.16). Тогда

$$
u_{1}=U\left(y, \nabla \psi_{0}(x)+\nabla u_{0}(x)\right)-\psi_{1}(x, y),
$$

где $U(\xi)$ является единственным (для каждого $\left.\xi \in \mathbb{R}^{n}\right)$ решением вариационного уравнения (1.18).

Используя это утверждение и выбирая $v_{1}=u_{1}$ в (1.16), получаем следующее макромасштабное осредненное (предельное для задачи (1.7)) вариационное неравенство для $u_{0} \in K_{0}$ :

$$
\begin{gathered}
\int_{\Omega} A\left(\nabla \psi_{0}+\nabla u_{0}\right)\left(\nabla v_{0}-\nabla u_{0}\right) d x \geqslant \int_{\Omega} f\left(v_{0}-u_{0}\right) d x \\
\forall v_{0} \in K_{0} \equiv\left\{v_{0} \in H_{0}^{1}(\Omega): v_{0} \geqslant 0 \text { п.в. в } \Omega\right\},
\end{gathered}
$$


где

$$
A(\xi)=\frac{1}{\theta} \int_{F} a\left(y, \xi+\nabla_{y} U(y, \xi)\right) d y, \quad \theta=\int_{F} 1 d y
$$

Посредством замены $\hat{u}_{0}=\psi_{0}+u_{0}, \hat{v}_{0}=\psi_{0}+v_{0}$ неравенство (1.19) эквивалентно следующему осредненному (предельному для задачи (1.5)) вариационному неравенству для $\hat{u}_{0} \in \widehat{K}_{0}$ :

$$
\begin{aligned}
& \int_{\Omega} A\left(\nabla \hat{u}_{0}\right)\left(\nabla \hat{v}_{0}-\nabla \hat{u}_{0}\right) d x \geqslant \int_{\Omega} f\left(\hat{v}_{0}-\hat{u}_{0}\right) d x \\
& \forall \hat{v}_{0} \in \widehat{K}_{0} \equiv\left\{\hat{v}_{0} \in H_{0}^{1}(\Omega): \hat{v}_{0} \geqslant \psi_{0} \text { п.в. в } \Omega\right\} .
\end{aligned}
$$

Отметим, что сушествование решения задачи (1.19) (и поэтому задачи (1.21)) вытекает из утверждения 1.2, а единственность - из строгой монотонности вектор-функции $A(\xi)$, которая следует из (1.6). Непосредственным следствием приведенных утверждений и леммы о компактности из [3, лемма 3.3] является следуюший результат о сходимости.

ТЕОРЕМА 1.5. Пусть $\hat{u}_{\varepsilon} \in K_{\varepsilon}$ является решением вариачионного неравенства (1.5) и выполнено предположение (1.11). Тогда

$$
\begin{gathered}
\chi_{\varepsilon} \hat{u}_{\varepsilon} \stackrel{2}{\longrightarrow} \chi \hat{u}_{0}, \quad \chi_{\varepsilon} \nabla \hat{u}_{\varepsilon} \stackrel{2}{\longrightarrow} \chi \nabla \hat{u}_{0}+\chi \nabla_{y} U\left(\nabla \hat{u}_{0}\right), \\
\left\|\hat{u}_{\varepsilon}-\hat{u}_{0}\right\|_{L^{2}\left(\Omega_{\varepsilon}\right)} \rightarrow 0, \quad \varepsilon \rightarrow 0,
\end{gathered}
$$

где $\hat{u}_{0} \in \widehat{K}_{0}$ является единственным решением осредненного вариачионного неравенства (1.21) и $U(\xi)$ является единственным (для каждого $\left.\xi \in \mathbb{R}^{n}\right)$ решением вариационного уравнения (1.18).

Вариационное неравенство (1.7) можно рассмотреть и в случае, когда множество $K$ заменено всем пространством $H_{D}^{1}\left(\Omega_{\varepsilon}\right)$. В этом случае известно [1], что такое вариационное неравенство эквивалентно вариационному уравнению для $u_{\varepsilon} \in$ $H_{D}^{1}\left(\Omega_{\varepsilon}\right)$ :

$$
\int_{\Omega_{\varepsilon}} a\left(\frac{x}{\varepsilon}, \nabla \psi_{\varepsilon}+\nabla u_{\varepsilon}\right) \nabla v d x=\int_{\Omega_{\varepsilon}} f v d x \quad \forall v \in H_{D}^{1}\left(\Omega_{\varepsilon}\right) .
$$

Также известно [1], что существует единственное (для фиксированного $\varepsilon$ ) решение вариационного уравнения (1.22) и что вариационное неравенство (1.19), в котором $K_{0}$ заменено на $H_{0}^{1}(\Omega)$, эквивалентно следующему вариационному уравнению для $u_{0} \in H_{0}^{1}(\Omega)$ :

$$
\int_{\Omega} A\left(\nabla \psi_{0}+\nabla u_{0}\right) \nabla v_{0} d x=\int_{\Omega} f v_{0} d x \quad \forall v_{0} \in H_{0}^{1}(\Omega) .
$$

Как следствие этих эквивалентностей и приведенных утверждений, где просто не учитывается неотрицательность рассматриваемых функций, получаем следуюший результат о сходимости решений задачи (1.22). 
ТЕОРемА 1.6. Пусть $u_{\varepsilon} \in H_{D}^{1}\left(\Omega_{\varepsilon}\right)$ является решением вариачионного уравнения (1.22) и выполнено предположение (1.11). Тогда

$$
\begin{gathered}
\chi_{\varepsilon} u_{\varepsilon} \stackrel{2}{\longrightarrow} \chi u_{0}, \quad \underset{\| u_{\varepsilon}}{\chi_{\varepsilon}} \nabla u_{\varepsilon} \stackrel{2}{\longrightarrow} \chi \nabla u_{0} \|_{L^{2}\left(\Omega_{\varepsilon}\right)} \rightarrow 0, \quad \chi \nabla_{y} U\left(\nabla \psi_{0}+\nabla u_{0}\right)-\chi \nabla_{y} \psi_{1}, \quad \varepsilon \rightarrow 0,
\end{gathered}
$$

əде $u_{0} \in H_{0}^{1}(\Omega)$ является единственным решением осредненной задачи (1.23), $U(\xi)$ является единственным (для каждого $\left.\xi \in \mathbb{R}^{n}\right)$ решением задачи (1.18), а $\psi_{0}$ и $\psi_{1}$ определены в утверждении 1.1 .

Вариационное уравнение (1.22) можно рассмотреть и в более общем случае. Пусть задана равномерно ограниченная последовательность функций $\left\{\widehat{\psi}_{\varepsilon}\right\} \subset$ $H^{1}\left(\Omega_{\varepsilon}\right)$. Тогда в соответствии с $\left[12\right.$, теорема 4.6] найдутся такие $\widehat{\psi}_{0}(x) \in H^{1}(\Omega)$ и $\widehat{\psi}_{1}(x, y) \in L^{2}\left(\Omega ; H_{P}^{1}(F) / \mathbb{R}\right)$, что (возможно, после выделения подпоследовательности при $\varepsilon \rightarrow 0$ )

$$
\chi_{\varepsilon} \widehat{\psi}_{\varepsilon} \stackrel{2}{\longrightarrow} \chi \widehat{\psi}_{0}, \quad \chi_{\varepsilon} \nabla \widehat{\psi}_{\varepsilon} \stackrel{2}{\longrightarrow} \chi \nabla \widehat{\psi}_{0}+\chi \nabla_{y} \widehat{\psi}_{1} .
$$

Пусть $\widehat{\psi}_{0}$ и $\widehat{\psi}_{1}$ определены однозначно (в этом случае соотношения (1.24) верны без выделения подпоследовательности) и выполнено равенство

$$
\lim _{\varepsilon \rightarrow 0}\left\|\nabla \widehat{\psi}_{\varepsilon}\right\|_{L^{2}\left(\Omega_{\varepsilon}\right)^{n}}=\left\|\nabla \widehat{\psi}_{0}+\nabla_{y} \widehat{\psi}_{1}\right\|_{L^{2}(\Omega \times F)^{n}} .
$$

Рассмотрим вариационное уравнение $(1.22)$, в котором $\psi_{\varepsilon}$ заменено $\widehat{\psi}_{\varepsilon}$. Известно [1], что посредством замены $\hat{u}_{\varepsilon}=\widehat{\psi}_{\varepsilon}+u_{\varepsilon}$ такой вариант вариационной задачи (1.22) является “обобщенной” постановкой задачи Дирихле-Неймана для $\hat{u}_{\varepsilon} \in H^{1}\left(\Omega_{\varepsilon}\right)$ :

$$
\begin{gathered}
-\operatorname{div}\left(a\left(\frac{x}{\varepsilon}, \nabla \hat{u}_{\varepsilon}\right)\right)=f \quad \text { в } \quad \Omega_{\varepsilon}, \quad \hat{u}_{\varepsilon}=\widehat{\psi}_{\varepsilon} \quad \text { на } \partial \Omega_{\varepsilon} \cap \partial \Omega \\
\left(\nu_{\varepsilon} \cdot a\left(\frac{x}{\varepsilon}, \nabla \hat{u}_{\varepsilon}\right)\right)=0 \text { на } S_{\varepsilon} .
\end{gathered}
$$

Также известно [1], что существует единственное (для фиксированного $\varepsilon$ ) решение задачи (1.26) (в обобщенной постановке $(1.22)$, где $\psi_{\varepsilon}$ заменено $\widehat{\psi}_{\varepsilon}$ и $u_{\varepsilon}=\hat{u}_{\varepsilon}-\widehat{\psi}_{\varepsilon}$ ). Из соотношений (1.4), (1.6) и предположений для исходных данных следует, что последовательности $\left\{\hat{u}_{\varepsilon}\right\}$ и $\left\{\nabla \hat{u}_{\varepsilon}\right\}$ решений задачи (1.26) и градиентов этих решений равномерно ограничены в $L^{2}\left(\Omega_{\varepsilon}\right)$ и $L^{2}\left(\Omega_{\varepsilon}\right)^{n}$ соответственно. Следствием приведенных утверждений является следующая теорема о сходимости решений задачи (1.26).

ТЕОРема 1.7. Пусть $\hat{u}_{\varepsilon} \in H^{1}\left(\Omega_{\varepsilon}\right)$ является решением задачи (1.26) и выполнены соотношения (1.24) и равенство (1.25). Тогда

$$
\begin{gathered}
\chi_{\varepsilon} \hat{u}_{\varepsilon} \stackrel{2}{\longrightarrow} \chi \hat{u}_{0}, \quad \chi_{\varepsilon} \nabla \hat{u}_{\varepsilon} \stackrel{2}{\longrightarrow} \chi \nabla \hat{u}_{0}+\chi \nabla_{y} U\left(\nabla \hat{u}_{0}\right), \\
\left\|\hat{u}_{\varepsilon}-\widehat{\psi}_{\varepsilon}-\hat{u}_{0}+\widehat{\psi}_{0}\right\|_{L^{2}\left(\Omega_{\varepsilon}\right)} \rightarrow 0, \quad \varepsilon \rightarrow 0,
\end{gathered}
$$


где $U(\xi)$ является единственным (для каждого $\left.\xi \in \mathbb{R}^{n}\right)$ решением задачи (1.18) и $\hat{u}_{0} \in H^{1}(\Omega)$ является единственным решением следующей задачи Дирихле для осредненного уравнения:

$$
-\operatorname{div}\left(A\left(\nabla \hat{u}_{0}\right)\right)=f \quad \text { в } \quad \Omega, \quad \hat{u}_{0}=\widehat{\psi}_{0} \quad \text { на } \quad \partial \Omega
$$

В случае, когда соответствующий строго монотонный эллиптический оператор в вариационном неравенстве (1.5) является потенциальным, это вариационное неравенство эквивалентно задаче минимизации на множестве $K_{\varepsilon}$. Поэтому естественно установить связь приведенных результатов с соответствующими результатами для задач минимизации. Таким образом, выберем $a(y, \xi)$ в задаче $(1.5)$ в следуюшем специальном виде:

$$
a(y, \xi)=\nabla_{\xi} b(y, \xi)
$$

где функция $b(y, \xi)$ является измеримой на $F$ для каждого $\xi \in \mathbb{R}^{n}$ и непрерывной относительно $\xi \in \mathbb{R}^{n}$ п.в. в $F$. Кроме того, предполагается, что для $a(y, \xi)$ выполнены неравенства (1.6). В этом случае известно [13], что функция $b(y, \xi)$ является строго выпуклой относительно $\xi \in \mathbb{R}^{n}$ п.в. в $F$ и вариационное неравенство $(1.7)$ эквивалентно (для фиксированного $\varepsilon$ ) задаче минимизации для $u_{\varepsilon} \in K$ :

$$
I_{\varepsilon}\left(u_{\varepsilon}\right)=\min _{v \in K} I_{\varepsilon}(v), \quad I_{\varepsilon}(v)=\int_{\Omega_{\varepsilon}}\left(b\left(\frac{x}{\varepsilon}, \nabla \psi_{\varepsilon}+\nabla v\right)-f v\right) d x
$$

Также известно [13], что вариационное уравнение (1.18) эквивалентно (для фиксированного $\left.\xi \in \mathbb{R}^{n}\right)$, если выполнено предположение (1.28), задаче минимизации для $U \in H_{P}^{1}(F) / \mathbb{R}$ :

$$
I_{\xi}(U)=\min _{V \in H_{P}^{1}(F) / \mathbb{R}} I_{\xi}(V), \quad I_{\xi}(V)=\int_{F} b\left(y, \xi+\nabla_{y} V\right) d y
$$

Рассмотрим также осредненную задачу минимизации для $u_{0} \in K_{0}$ :

$$
I_{0}\left(u_{0}\right)=\min _{v_{0} \in K_{0}} I_{0}\left(v_{0}\right), \quad I_{0}\left(v_{0}\right)=\int_{\Omega}\left(B\left(\nabla \psi_{0}+\nabla v_{0}\right)-f v_{0}\right) d x
$$

где

$$
B(\xi)=\left(\theta^{-1}\right) \int_{F} b\left(y, \xi+\nabla_{y} U(y, \xi)\right) d y
$$

и $U(y, \xi)$ является решением задачи минимизации (1.30) для каждого $\xi \in \mathbb{R}^{n}$.

УТВЕРЖДЕНИЕ 1.8. Существует единственное решение $u_{0} \in K_{0}$ задачи минимизации (1.31), и эта задача эквивалентна осредненному вариачионному неравенству (1.19), если выполнено равенство (1.28).

Отметим, что

$$
\nabla_{\xi} B(\xi)=\theta^{-1} \int_{F} \nabla_{\xi} b\left(y, \xi+\nabla_{y} U(\xi)\right)\left(E+\nabla_{\xi} \nabla_{y} U(\xi)\right) d y
$$

по крайней мере формально, и поэтому утверждение 1.8 не является прямым следствием из известных утверждений, поскольку равенство $A(\xi)=\nabla_{\xi} B(\xi)$ не следует непосредственно из (1.20) и (1.28). 
Рассмотрим, кроме того, следуюшую двухмасштабную задачу минимизации для $\left(u_{0}, u_{1}\right) \in K_{2}$ :

$$
\begin{gathered}
I_{2}\left(u_{0}, u_{1}\right)=\min _{\left(v_{0}, v_{1}\right) \in K_{2}} I_{2}\left(v_{0}, v_{1}\right), \\
I_{2}\left(v_{0}, v_{1}\right)=\int_{\Omega} \int_{F}\left(b\left(y, \nabla \psi_{0}+\nabla_{y} \psi_{1}+\nabla v_{0}+\nabla_{y} v_{1}\right)-f v_{0}\right) d y d x .
\end{gathered}
$$

УТВЕРЖДЕНИЕ 1.9. Существует единственное решение $\left(u_{0}, u_{1}\right) \in K_{2}$ задачи минимизачии (1.32), и эта задача әквивалентна двухмаситабному вариационному неравенству (1.16), если выполнено равенство (1.28).

Таким образом, эквивалентность (при $\left.a(y, \xi)=\nabla_{\xi} b(y, \xi)\right)$ задачи минимизации (1.29) и рассмотренного в настоящей работе вариационного неравенства (1.7) (эквивалентного вариационному неравенству (1.5)) сохраняется при макромасштабном осреднении и двухмасштабном осреднении, а утверждение о сходимости решений задачи минимизации (1.29) следует из теоремы 1.5. Аналогично, задача минимизации $(1.29)$, в которой $K$ заменено на $H_{D}^{1}\left(\Omega_{\varepsilon}\right)$, и задача минимизации $(1.31)$, в которой $K_{0}$ заменено на $H_{0}^{1}(\Omega)$, эквивалентны вариационным уравнениям (1.22) и (1.23) соответственно, и утверждение о сходимости решений в этом случае следует из теоремы 1.6 (и теоремы 1.7, соответственно, при более общих предположениях (1.24) и (1.25)).

Проверяется, что предположение (1.11) выполнено, если

$$
\psi_{\varepsilon}=\psi_{\varepsilon}^{0}+\varepsilon \psi_{1}\left(x, \frac{x}{\varepsilon}\right),
$$

где $\psi_{1}(x, y) \in L^{2}\left(\Omega ; L_{P}^{\infty}(F)\right), \quad \nabla_{y} \psi_{1}(x, y), \nabla_{x} \psi_{1}(x, y) \in L^{2}\left(\Omega ; L_{P}^{\infty}(F)^{n}\right), \quad \psi_{\varepsilon}^{0} \in$ $H_{0}^{1}(\Omega)$ и $\psi_{\varepsilon}^{0} \rightarrow \psi_{0}$ сильно в $H_{0}^{1}(\Omega)$ при $\varepsilon \rightarrow 0$. В таком смысле равенство $(1.11)$ является условием регулярности на (равномерно ограниченную) последовательность $\left\{\psi_{\varepsilon}\right\} \subset H_{D}^{1}\left(\Omega_{\varepsilon}\right)$ в задаче (1.5). Появление пространства $L^{2}\left(\Omega ; L_{P}^{\infty}(F)\right)$ связано с выполнением равенства

$$
\lim _{\varepsilon \rightarrow 0} \int_{\Omega} \varphi\left(x, \frac{x}{\varepsilon}\right)^{2} d x=\int_{\Omega} \int_{Y} \varphi(x, y)^{2} d y d x,
$$

доказанного для $\varphi \in L_{P}^{2}(Y ; C(\bar{\Omega}))$ и для $\varphi \in L^{2}\left(\Omega ; C_{P}(Y)\right)$ в $[9]$, а для $\varphi \in$ $L^{2}\left(\Omega ; L_{P}^{\infty}(Y)\right)$ - в [16]. Известны примеры функций $\varphi \in C\left(\bar{\Omega} ; L_{P}^{2}(Y)\right)$, для которых не выполнено равенство (1.34) (см. [9, утверждение 5.8]). Более того, для $\varphi \in C\left(\bar{\Omega} ; L_{P}^{2}(Y)\right)$ выражение $\varphi(x, x / \varepsilon)$ может не иметь корректного смысла, поскольку это выражение является "следом" на $n$-плоскости $x=x, y=\varepsilon^{-1} x$ (зависяшей от $\varepsilon$ ) в пространстве переменных $x, y$ и необходима некоторая регулярность по $y$ для определения такого "следа". Условие $\varphi(x, y) \in L^{2}\left(\Omega ; L_{P}^{\infty}(Y)\right)$ является в некотором смысле минимальным (из известных условий), при выполнении которого выражение $\varphi(x, x / \varepsilon)$ определено и выполнено (1.34) в соответствии с [16].

Непосредственно из определений следует, что для $\varphi \in L^{2}\left(\Omega ; L_{P}^{\infty}(F)\right)$ (или $\varphi \in$ $\left.L_{P}^{2}(F ; C(\bar{\Omega}))\right)$ продолжение $\chi \varphi$ принадлежит $L^{2}\left(\Omega ; L_{P}^{\infty}(Y)\right) \quad(\chi \varphi$ принадлежит $\left.L_{P}^{2}(Y ; C(\bar{\Omega}))\right)$. Таким образом, для перфорированной области $\Omega_{\varepsilon}$ и, например, $\varphi \in L^{2}\left(\Omega ; L_{P}^{\infty}(F)\right)$ равенство (1.34) можно записать в виде

$$
\lim _{\varepsilon \rightarrow 0} \int_{\Omega_{\varepsilon}} \varphi\left(x, \frac{x}{\varepsilon}\right)^{2} d x=\lim _{\varepsilon \rightarrow 0} \int_{\Omega} \chi_{\varepsilon} \varphi\left(x, \frac{x}{\varepsilon}\right)^{2} d x=\int_{\Omega} \int_{F} \varphi(x, y)^{2} d y d x .
$$


В работе [9] было отмечено также, что равенство (1.34) в определенном смысле характеризует тестовые функции $\varphi(x, y)$, которые допустимо выбирать в определении (1.10) с сохранением компактности двухмасштабной сходимости (из этой компактности для равномерно ограниченных последовательностей из $L^{2}(\Omega)$ и следует утверждение 1.1). В оригинальном определении двухмасштабной сходимости из [8] тестовые функции выбирались из $C_{c}\left(\Omega ; C_{P}(Y)\right)$ и компактность доказана в этом случае. Однако при переходе к пределу в неравенстве (1.14) с использованием только теоремы компактности из [8] необходимы дополнительные предположения о непрерьвности вектор-функции $a(y, \xi)$ по $y$. Таким образом, использование теоремы компактности из [9] приводит к некоторому ослаблению условий регулярности на исходные данные.

ЗАмЕчАниЕ 1.10. Задача $(1.22)$ с линейной по $\xi$ вектор-функцией $a(y, \xi)$ и $\psi_{\varepsilon}=0$ рассмотрена в работе [3], где использовались метод компактности и энергетический метод для доказательства теоремы о сходимости, и в работе [9], где использовался метод двухмасштабной сходимости. Задачи минимизации без ограничений в перфорированных областях рассматривались также методами Г-сходимости, например, в [14]. В этих работах можно найти и более подробную библиографию.

ЗАмЕчАниЕ 1.11. Задача минимизации (1.29) с квадратичной по $\xi$ функцией $b(y, \xi)$ и $\psi_{\varepsilon}=0$ рассматривалась в статье [15] при значительно более обших предположениях о регулярности границы перфорированной области $\Omega_{\varepsilon}$. Впрочем, корректность такой задачи минимизации и корректность определения следа $\left.u_{\varepsilon}\right|_{S_{\varepsilon}}$ не ясны и не доказаны в [15], поскольку в предположениях этой статьи граница $S_{\varepsilon}$ может не быть даже непрерьвной и компактной (при фиксированном $\varepsilon$ ). Кроме того, теорема о сходимости $[15$, теорема 2.2$]$ доказана в этой статье фактически только в предположении, что решения рассматриваемой задачи минимизации $[15,(1.1)]$ являются гладкими, что не верно в общем случае даже для регулярных границ и фиксированного $\varepsilon$. Аналогичные предположения (о регулярности границы перфорированной области $\Omega_{\varepsilon}$ ) и методы (доказательства теорем сходимости для гладких решений) использовались в статьях [17] и [18] для рассмотрения задач минимизации без ограничений и вариационных задач с монотонными операторами в перфорированных областях.

ЗАмечАниЕ 1.12. Используемые здесь методы применимы к вариационным неравенствам для задач с препятствием в ограниченных областях [19]. Кроме того, эти методы применимы и к вариационным неравенствам для задач с препятствием в перфорированных областях. Используя обозначения и предположения для задачи (1.5), приведем точные утверждения для таких задач с препятствием в периодически перфорированных областях, определяемых равенством (1.1).

Рассмотрим следующее вариационное неравенство для $\hat{u}_{\varepsilon} \in K_{\varepsilon}^{o}$ :

$$
\begin{gathered}
\int_{\Omega_{\varepsilon}} a\left(\frac{x}{\varepsilon}, \nabla \hat{u}_{\varepsilon}\right)\left(\nabla \hat{v}-\nabla \hat{u}_{\varepsilon}\right) d x \geqslant \int_{\Omega_{\varepsilon}} f\left(\hat{v}-\hat{u}_{\varepsilon}\right) d x \\
\forall \hat{v} \in K_{\varepsilon}^{o} \equiv\left\{\hat{v} \in H_{D}^{1}\left(\Omega_{\varepsilon}\right): \hat{v} \geqslant \psi_{\varepsilon} \text { п.в. в } \Omega_{\varepsilon}\right\},
\end{gathered}
$$

где $f \in L^{2}(\Omega)$ и последовательность $\left\{\psi_{\varepsilon}\right\} \subset H_{D}^{1}\left(\Omega_{\varepsilon}\right)$ является равномерно ограниченной относительно $\varepsilon$. Известно [1], что существует единственное (для фиксированного $\varepsilon$ ) решение вариационного неравенства (1.36) и посредством замены 
$u_{\varepsilon}=\hat{u}_{\varepsilon}-\psi_{\varepsilon}, v=\hat{v}-\psi_{\varepsilon}$ это неравенство эквивалентно вариационному неравенству для $u_{\varepsilon} \in K^{o}$ :

$$
\begin{gathered}
\int_{\Omega_{\varepsilon}} a\left(\frac{x}{\varepsilon}, \nabla \psi_{\varepsilon}+\nabla u_{\varepsilon}\right)\left(\nabla v-\nabla u_{\varepsilon}\right) d x \geqslant \int_{\Omega_{\varepsilon}} f\left(v-u_{\varepsilon}\right) d x \\
\forall v \in K^{o} \equiv\left\{v \in H_{D}^{1}\left(\Omega_{\varepsilon}\right): v \geqslant 0 \text { п.в. в } \Omega_{\varepsilon}\right\},
\end{gathered}
$$

где множество $K^{o}$ является замкнутым выпуклым конусом с вершиной в нуле (подробности можно найти, например, в [1] и [20]).

Также известно [1], [20], что вариационное неравенство (1.37) эквивалентно следуюшему вариационному неравенству для $u_{\varepsilon} \in K^{o}$ :

$$
\int_{\Omega_{\varepsilon}} a\left(\frac{x}{\varepsilon}, \nabla \psi_{\varepsilon}+\nabla v\right)\left(\nabla v-\nabla u_{\varepsilon}\right) d x \geqslant \int_{\Omega_{\varepsilon}} f\left(v-u_{\varepsilon}\right) d x \quad \forall v \in K^{o} .
$$

Как и для задачи (1.7), из соотношений (1.4), (1.6) и предположений для исходных данных вытекает следующее утверждение.

УТВЕРЖДЕНИЕ 1.13. Пусть $u_{\varepsilon}(x) \in H_{D}^{1}\left(\Omega_{\varepsilon}\right)$ является решением вариаиионного неравенства (1.37). Тогда найдутся такие $\psi_{0}(x), u_{0}(x) \in H_{0}^{1}(\Omega)$ и $\psi_{1}(x, y), u_{1}(x, y) \in L^{2}\left(\Omega ; H_{P}^{1}(F) / \mathbb{R}\right)$, что (возможсно, после вьделения подпоследовательностей при $\varepsilon \rightarrow 0$ )

$$
\begin{array}{cc}
\chi_{\varepsilon} \psi_{\varepsilon} \stackrel{2}{\longrightarrow} \chi \psi_{0}, & \chi_{\varepsilon} u_{\varepsilon} \stackrel{2}{\longrightarrow} \chi u_{0}, \\
\chi_{\varepsilon} \nabla \psi_{\varepsilon} \stackrel{2}{\longrightarrow} \chi \nabla \psi_{0}+\chi \nabla_{y} \psi_{1}, & \chi_{\varepsilon} \nabla u_{\varepsilon} \stackrel{2}{\longrightarrow} \chi \nabla u_{0}+\chi \nabla_{y} u_{1}
\end{array}
$$

$u u_{0} \geqslant 0$ n.в. $в \Omega$.

Далее предполагается, что $\psi_{0}$ и $\psi_{1}$ из утверждения 1.13 определены однозначно и выполнено равенство (1.11). Кроме того, в соответствии с утверждением 1.13 множество $K_{2}$ содержит всевозможные двухмасштабные пределы решений задачи (1.37).

Предположим, что $v_{0}, \psi_{0} \in C_{0}^{\infty}(\Omega)$ и $v_{1}, \psi_{1} \in C_{0}^{\infty}\left(\Omega ; C_{P}^{\infty}(Y) / \chi \mathbb{R}\right)$ при $v_{0} \geqslant 0$ в $\Omega$. Тогда $\left(v_{0}, v_{1}\right) \in K_{2}$ и можно выбрать тестовую функцию $v=v_{0}(x)+$ $\varepsilon v_{1}(x, x / \varepsilon)+\varepsilon \delta e_{\varepsilon}$ в неравенстве из (1.38) (см. (1.14)). Следовательно, переходя к пределу в этом неравенстве, заключаем, что $u_{0}$ и $u_{1}$, определенные в утверждении 1.13, удовлетворяют неравенству (1.15) для всех $\left(v_{0}, v_{1}\right) \in K_{2}$. Проверяется также, что неравенство (1.15) выполнено и для произвольных $\psi_{0}$ и $\psi_{1}$, определенных в утверждении 1.13 и удовлетворяющих предположению (1.11).

Кроме того, выполняются и утверждения, аналогичные утверждениям 1.2-1.4. Таким образом, осредненное вариационное неравенство (1.19) является предельной задачей и для вариационного неравенства (1.37), а осредненное вариационное неравенство (1.21) является предельной задачей и для вариационного неравенства (1.36). Непосредственным следствием приведенных утверждений и леммы о компактности из [3] является следующий результат о сходимости решений задачи (1.36).

ТЕОРЕМА 1.14. Пусть $\hat{u}_{\varepsilon} \in K_{\varepsilon}^{o}$ является решением вариационного неравенства (1.36) и выполнено предположение (1.11). Тогда

$$
\begin{gathered}
\chi_{\varepsilon} \hat{u}_{\varepsilon} \stackrel{2}{\longrightarrow} \chi \hat{u}_{0}, \quad \chi_{\varepsilon} \nabla \hat{u}_{\varepsilon} \stackrel{2}{\longrightarrow} \chi \nabla \hat{u}_{0}+\chi \nabla_{y} U\left(\nabla \hat{u}_{0}\right), \\
\left\|\hat{u}_{\varepsilon}-\hat{u}_{0}\right\|_{L^{2}\left(\Omega_{\varepsilon}\right)} \rightarrow 0, \quad \varepsilon \rightarrow 0,
\end{gathered}
$$


где $\hat{u}_{0} \in \widehat{K}_{0}$ является единственным решением осредненного вариационного неравенства (1.21) и $U(\xi)$ является единственным (для каждого $\left.\xi \in \mathbb{R}^{n}\right)$ решением вариащионного уравнения (1.18).

В случае выполнения равенства (1.28) также известно [13], что вариационное неравенство (1.37) эквивалентно (для фиксированного $\varepsilon$ ) задаче минимизации для $u_{\varepsilon} \in K^{o}$ :

$$
I_{\varepsilon}\left(u_{\varepsilon}\right)=\min _{v \in K^{o}} I_{\varepsilon}(v), \quad I_{\varepsilon}(v)=\int_{\Omega_{\varepsilon}}\left(b\left(\frac{x}{\varepsilon}, \nabla \psi_{\varepsilon}+\nabla v\right)-f v\right) d x
$$

поэтому утверждение о сходимости решений этой задачи минимизации к решению задачи минимизации (1.31) является следствием из теоремы 1.14 (и утверждения 1.8).

Таким образом, решения вариационного неравенства (1.5) (для задачи с условием Синьорини) “достаточно близки” к решениям вариационного неравенства (1.36) (для задачи с препятствием), поскольку эти задачи при совпадаюших исходных данных имеют один и тот же предел при $\varepsilon \rightarrow 0$, определяемый решением осредненного вариационного неравенства (1.21) (для задачи с препятствием). Такое свойство решений задачи (1.5) достаточно естественно, поскольку условия Синьорини задаются на достаточно "массивном" множестве $S_{\varepsilon}$, что вполне иллюстрируется следуюшим примером. Используя обозначения и предположения для задачи (1.5), рассмотрим вариационное уравнение для $u_{\varepsilon} \in H_{0}^{1}\left(\Omega_{\varepsilon}\right)$ :

$$
\begin{gathered}
\int_{\Omega_{\varepsilon}} a\left(\frac{x}{\varepsilon}, \nabla u_{\varepsilon}\right) \nabla v d x=\int_{\Omega_{\varepsilon}} f v d x \\
\forall v \in H_{0}^{1}\left(\Omega_{\varepsilon}\right) \equiv\left\{v \in H_{D}^{1}\left(\Omega_{\varepsilon}\right): v=0 \text { на } S_{\varepsilon}\right\} .
\end{gathered}
$$

Известно [1], что сушествует единственное (для фиксированного $\varepsilon$ ) решение этого уравнения. Кроме того, из (1.6) и неравенства Фридрихса [21, прилож., лемма 1] следует, что $\left\|u_{\varepsilon}\right\|_{L^{2}\left(\Omega_{\varepsilon}\right)} \leqslant C \varepsilon^{2}$ и $\left\|\nabla u_{\varepsilon}\right\|_{L^{2}\left(\Omega_{\varepsilon}\right)} \leqslant C \varepsilon$, где постоянная $C$ не зависит от $\varepsilon$. Таким образом, равенство $\left.u_{\varepsilon}\right|_{S_{\varepsilon}}=0$ “достаточно близко" к равенству $u_{\varepsilon}=0$ для решений вариационных уравнений с монотонным оператором в перфорированной области $\Omega_{\varepsilon}$.

В соответствии с [1], [20] решение $\hat{u}_{\varepsilon} \in K_{\varepsilon}^{o}$ вариационного неравенства (1.36) характеризуется также следующими условиями (возможно, при дополнительных условиях регулярности на исходные данные):

$$
\begin{gathered}
-\operatorname{div}\left(a\left(\frac{x}{\varepsilon}, \nabla \hat{u}_{\varepsilon}\right)\right)=f \quad \text { в } \Omega_{\varepsilon}^{+} \equiv\left\{x \in \Omega_{\varepsilon}: \hat{u}_{\varepsilon}(x)>\psi_{\varepsilon}(x)\right\}, \\
\hat{u}_{\varepsilon}=\psi_{\varepsilon} \quad \text { в } \Omega_{\varepsilon}^{0} \equiv \Omega_{\varepsilon} \backslash \Omega_{\varepsilon}^{+} \\
\hat{u}_{\varepsilon}=\psi_{\varepsilon} \quad \text { и }\left(\nu_{\varepsilon} \cdot a\left(\frac{x}{\varepsilon}, \nabla \hat{u}_{\varepsilon}\right)\right)=0 \text { на } \partial \Omega_{\varepsilon}^{+} \\
\hat{u}_{\varepsilon}=0 \text { на } \partial \Omega_{\varepsilon} \cap \partial \Omega \\
\left(\nu_{\varepsilon} \cdot a\left(\frac{x}{\varepsilon}, \nabla \hat{u}_{\varepsilon}\right)\right)=0 \text { на } S_{\varepsilon} .
\end{gathered}
$$

В силу условий этой задачи область $\Omega_{\varepsilon}$ допускает разбиение на две части: $\Omega_{\varepsilon}^{0}=\left\{\hat{u}_{\varepsilon}=\psi_{\varepsilon}\right\}$ и $\Omega_{\varepsilon}^{+}=\left\{\hat{u}_{\varepsilon}>\psi_{\varepsilon}\right\}$, и это разбиение (определяемое "свободной" 
гранищей) является одним из неизвестных задачи (см., например, [1], [20]). Таким образом, задача (1.39) может быть интерпретирована как модель для установившейся нелинейной диффузии (или теплопроводности) в пористой среде, часть которой является “застойной” (или имеет предписанное распределение температуры), а часть "активна", на этой части собственно и моделируется процесс нелинейной диффузии (или процесс распространения тепла).

Соответственно, решение $\hat{u}_{\varepsilon} \in \widehat{K}_{0}$ осредненного (предельного для вариационных неравенств (1.5) и (1.36)) вариационного неравенства (1.21) характеризуется также следуюшими условиями:

$$
\begin{gathered}
-\operatorname{div}\left(A\left(\nabla \hat{u}_{0}\right)\right)=f \quad \text { в } \Omega^{+} \equiv\left\{x \in \Omega: \hat{u}_{0}(x)>\psi_{0}(x)\right\}, \\
\hat{u}_{0}=\psi_{0} \quad \text { в } \Omega^{0} \equiv \Omega \backslash \Omega^{+}, \\
\hat{u}_{0}=\psi_{0} \text { и }\left(\nu \cdot A\left(\nabla \hat{u}_{0}\right)\right)=0 \text { на } \partial \Omega^{+}, \\
\hat{u}_{0}=0 \text { на } \partial \Omega,
\end{gathered}
$$

где $\nu$ - вектор внешней нормали к гранище $\partial \Omega^{+}$. Таким образом, решения задач (1.9) и (1.39) “достаточно близки” к решениям этой задачи, имеющей “застойную" $\Omega^{0}$ и “активную" $\Omega^{+}$зоны диффузии (или теплопроводности).

ЗАмЕчАниЕ 1.15. Задача об осреднении вариационных неравенств в ограниченных (не перфорированных) областях ставилась в книге [22], где методом "штрафа" было рассмотрено нестационарное вариационное неравенство для задачи с препятствием для линейного оператора с быстроосциллирующими коэффициентами в ограниченной области. Двухмасштабные предельные задачи для уравнений со строго монотонными операторами в ограниченных областях и задач минимизации без ограничений получены в [9]. Однако утверждения о связи этих двухмасштабных задач с ранее полученными макромасштабными задачами установлены в [9] только для линейного случая. Отметим также, что двухмасштабные предельные задачи впервые получили Ж.-Л. Лионс и Е. Санчес-Паленсия на основе равенств, аналогичных (1.34), например, в [23], но без доказательства соответствующих утверждений о сходимости решений. Обшие вопросы сходимости решений задач минимизации с препятствием, зависящих от параметра, рассматривались методами Г-сходимости во многих работах (см., например, [24] и [25]).

\section{§2. Доказательства}

Далее через $C$ обозначены (возможно, различные) постоянные, не зависяшие от положительного параметра $\varepsilon$. Кроме того, для заданной 1-периодической на $Y$ функции $w(y)$ (возможно, зависяшей и от других переменных) через $w^{\varepsilon}=w(x / \varepsilon)$ обозначено $\varepsilon$-периодическое продолжение этой функции на $\Omega$. Более точно, $w(y)$ продолжается как 1-периодическая функция на $\mathbb{R}^{n}$, и выбирается ограничение полученной функции при $y=x / \varepsilon$ на $\Omega$, которое и обозначается $w^{\varepsilon}$.

Известно, что множество $C_{0}^{\infty}(\Omega)$ плотно в сепарабельных пространствах $L^{2}(\Omega)$ и $H_{0}^{1}(\Omega)$ (см., например, $[26$, гл. $\left.2, \S 2]\right)$. Проверим, что множество $C_{0}^{\infty}(\Omega$; $\left.C_{P}^{\infty}(Y) / \chi \mathbb{R}\right)$ плотно в $L^{2}\left(\Omega ; H_{P}^{1}(F) / \mathbb{R}\right) . \quad$ С точностью до изоморфизма (см. [10, гл. 1, замечание 1.4]) пространство $H_{P}^{1}(F) / \mathbb{R}$ можно рассматривать как подпространство в $H_{P}^{1}(F)$, состоящее из функций $\varphi \in H_{P}^{1}(F)$, удовлетворяющих равен- 
ству $\int_{F} \varphi d y=0$ (из которого следует замкнутость $H_{P}^{1}(F) / \mathbb{R}$ в $H_{P}^{1}(F)$ ). Из (локальной) липшицевости $F$ следует, что множество $C_{P}^{\infty}(Y)$, ограниченное на $F$, плотно в сепарабельном пространстве $H_{P}^{1}(F)$ (см., например, [26]). Точнее, это утверждение известно для липшицевых областей, а для подмногообразий с липшицевыми границами это проверяется с помощью подходящей локализации. Следовательно, множество $C_{P}^{\infty}(Y) / \chi \mathbb{R}$ плотно в $H_{P}^{1}(F) / \mathbb{R}$, поскольку $H_{P}^{1}(F) / \mathbb{R}$ замкнуто в $H_{P}^{1}(F)$. В соответствии с $\left[27\right.$, теорема II.10] пространство $L^{2}\left(\Omega ; H_{P}^{1}(F) / \mathbb{R}\right)$ изоморфно $L^{2}(\Omega) \otimes\left(H_{P}^{1}(F) / \mathbb{R}\right)$ и конечные линейные комбинации произведений $\varphi_{1}(x) \varphi_{2}(y)$ для $\varphi_{1} \in L^{2}(\Omega), \varphi_{2} \in H_{P}^{1}(F) / \mathbb{R}$ плотны в этом пространстве, что доказывает плотность $C_{0}^{\infty}\left(\Omega ; C_{P}^{\infty}(Y) / \chi \mathbb{R}\right)$ в $L^{2}\left(\Omega ; H_{P}^{1}(F) / \mathbb{R}\right)$.

Из последнего утверждения и неравенства треугольника следует, что для $\left(v_{0}, v_{1}\right) \in H_{2}$ можно найти такие последовательности $\left\{v_{0}^{i}\right\} \subset C_{0}^{\infty}(\Omega)$ и $\left\{v_{1}^{i}\right\} \subset$ $C_{0}^{\infty}\left(\Omega ; C_{P}^{\infty}(Y) / \chi \mathbb{R}\right)$, что

$$
\left\|\left(v_{0}-v_{0}^{i}, v_{1}-v_{1}^{i}\right)\right\|_{2} \rightarrow 0, \quad i \rightarrow \infty .
$$

Отметим также, что соотношение (2.1) при $\left\{v_{0}^{i}\right\} \subset C_{0}^{\infty}(\Omega) \cap K_{0}$ выполняется и в случае, когда $\left(v_{0}, v_{1}\right) \in K_{2}$, поскольку множество $C_{0}^{\infty}(\Omega) \cap K_{0}$ (см. (1.19)) плотно в $K_{0}$ в соответствии с $[4, \S 3]$.

Из липшицевости $F$ следует также неравенство Пуанкаре

$$
\|\varphi\|_{L^{2}(F)}^{2} \leqslant C\left\|\nabla_{y} \varphi\right\|_{L^{2}(F)^{n}}^{2}, \quad \varphi \in H_{P}^{1}(F) / \mathbb{R}
$$

(см., например, $[10$, гл. 1, предложение 1.2]). Тогда непосредственно из определений заключаем, что также выполнено неравенство

$$
\left\|v_{1}\right\|_{L^{2}\left(\Omega ; L^{2}(F)\right)}^{2} \leqslant C\left\|\nabla_{y} v_{1}\right\|_{L^{2}\left(\Omega ; L^{2}(F)^{n}\right)}^{2}, \quad v_{1} \in L^{2}\left(\Omega ; H_{P}^{1}(F) / \mathbb{R}\right) .
$$

ДОКАЗАТЕЛЬСТВО УТВЕРЖДЕНИЯ 1.1. ВЫбирая $v=0$ в неравенстве из (1.7) и добавляя соответствуюшие интегралы, имеем

$$
\begin{aligned}
\alpha\left\|\nabla u_{\varepsilon}+\nabla \psi_{\varepsilon}\right\|_{L^{2}\left(\Omega_{\varepsilon}\right)^{n}} \leqslant & C\|f\|_{L^{2}(\Omega)}\left\|\nabla u_{\varepsilon}\right\|_{L^{2}\left(\Omega_{\varepsilon}\right)^{n}} \\
& +\beta\left\|\nabla u_{\varepsilon}+\nabla \psi_{\varepsilon}\right\|_{L^{2}\left(\Omega_{\varepsilon}\right)^{n}}\left\|\nabla \psi_{\varepsilon}\right\|_{L^{2}\left(\Omega_{\varepsilon}\right)^{n}},
\end{aligned}
$$

где учтены неравенства Гёльдера и соотношения (1.4), (1.6). В этом случае для получения оценки

$$
\left\|\nabla u_{\varepsilon}\right\|_{L^{2}\left(\Omega_{\varepsilon}\right)^{n}}^{2} \leqslant C\left(\|f\|_{L^{2}(\Omega)}^{2}+\left\|\nabla \psi_{\varepsilon}\right\|_{L^{2}\left(\Omega_{\varepsilon}\right)^{n}}^{2}\right)
$$

достаточно воспользоваться неравенством Юнга $a b \leqslant(\sigma / 2) a^{2}+b^{2} /(2 \sigma)$ и неравенством треугольника $\left\|\nabla u_{\varepsilon}\right\|-\left\|\nabla \psi_{\varepsilon}\right\| \leqslant\left\|\nabla u_{\varepsilon}+\nabla \psi_{\varepsilon}\right\|$.

Таким образом, последовательности $\left\{u_{\varepsilon}\right\}$ и $\left\{\nabla u_{\varepsilon}\right\}$ решений задачи (1.7) и градиентов этих решений равномерно ограничены в $L^{2}\left(\Omega_{\varepsilon}\right)$ и $L^{2}\left(\Omega_{\varepsilon}\right)^{n}$ соответственно. Тогда утверждения о сходимости из утверждения 1.1 следуют из результатов работы [9, доказательство теоремы 2.9], а утверждение о неотрицательности $u_{0}$ доказано фактически в $[15$, лемма 4.2$]$, что завершает доказательство утверждения 1.1.

Отметим, что лемма 4.2 из [15] сформулирована при значительно более обших предположениях о регулярности гранишы $S_{\varepsilon}$, но в доказательстве этой леммы используется разрешимость неоднородной 1-периодической задачи Неймана $[15,(4.9)]$. Однако разрешимость такой задачи известна при условии липшицевости границы $S$ (см., например, [6]). При этом условии регулярности определено 
и понятие граничного интеграла по $S$ (например, в соответствии с [2, гл. $1, \S 1.6]$ ), также используемое в доказательстве лемма 4.2 из [15].

Лемма 2.1. Пусть $\psi_{0}, u_{0} \in H_{0}^{1}(\Omega)$ u $\psi_{1}, u_{1} \in L^{2}\left(\Omega ; H_{P}^{1}(F) / \mathbb{R}\right)$ onределены в утверждении 1.1. Тогда $\left(u_{0}, u_{1}\right) \in K_{2}$ удовлетворяет вариационному неравенству (1.15) для каждого $\left(v_{0}, v_{1}\right) \in K_{2}$.

ДокАЗАТЕЛЬСтво. Пусть $w(x, y) \in C_{0}^{\infty}\left(\Omega ; C_{P}^{\infty}(Y)\right)$. Непосредственно из определения (1.10) следует, что

$$
w\left(x, \frac{x}{\varepsilon}\right) \stackrel{2}{\longrightarrow} w(x, y), \quad \chi_{\varepsilon} w\left(x, \frac{x}{\varepsilon}\right) \stackrel{2}{\longrightarrow} \chi w(x, y)
$$

(см. [8], [9]). Хорошо известна также следуюшая формула дифференцирования быстроосциллируюших функций:

$$
\nabla\left(w\left(x, \frac{x}{\varepsilon}\right)\right)=\left(\varepsilon^{-1} \nabla_{y} w(x, y)+\nabla_{x} w(x, y)\right), \quad y=\frac{x}{\varepsilon} .
$$

Выберем в неравенстве из (1.8) тестовую функцию

$$
v_{\varepsilon}=v_{0}(x)+\varepsilon v_{1}\left(x, \frac{x}{\varepsilon}\right)+\varepsilon \delta e_{\varepsilon},
$$

где $v_{0} \in C_{0}^{\infty}(\Omega), v_{0} \geqslant 0$ в $\Omega, v_{1} \in C_{0}^{\infty}\left(\Omega ; C_{P}^{\infty}(Y) / \chi \mathbb{R}\right)$ и подходящее $e_{\varepsilon}$ построено в $[11$, гл. $4, \S 1]$, где для липшицевой границы $\partial \Omega$ следует также учесть теорему Радемахера (см., например, [20]). Тогда, учитывая (1.6), неравенство из (1.8) можно представить в виде

$$
\int_{\Omega_{\varepsilon}} a\left(\frac{x}{\varepsilon}, \nabla \psi_{\varepsilon}+\nabla v_{0}+\nabla_{y} v_{1}^{\varepsilon}\right)\left(\nabla v_{0}+\nabla_{y} v_{1}^{\varepsilon}-\nabla u_{\varepsilon}\right) d x \geqslant \int_{\Omega_{\varepsilon}} f\left(v_{0}-u_{\varepsilon}\right) d x+\sigma_{\varepsilon},
$$

где $\lim _{\varepsilon \rightarrow 0} \sigma_{\varepsilon}=0$, поскольку $\lim _{\varepsilon \rightarrow 0}\left\|\nabla v_{\varepsilon}-\nabla v_{0}-\nabla_{y} v_{1}^{\varepsilon}\right\|_{L^{2}\left(\Omega_{\varepsilon}\right)^{n}}=0$ в силу $(2.6)$ и $(2.7)$.

В соответствии с (2.1) найдутся такие последовательности $\left\{\psi_{0}^{i}\right\} \subset C_{0}^{\infty}(\Omega)$ и $\left\{\psi_{1}^{i}\right\} \subset C_{0}^{\infty}\left(\Omega ; C_{P}^{\infty}(Y) / \chi \mathbb{R}\right)$, что

$$
\lim _{i \rightarrow \infty}\left\|\nabla \psi_{0}+\nabla_{y} \psi_{1}-\nabla \psi_{0}^{i}-\nabla_{y} \psi_{1}^{i}\right\|_{L^{2}(\Omega \times F)^{n}}=0 .
$$

Кроме того, из (1.11) следует, что

$$
\lim _{i \rightarrow \infty} \lim _{\varepsilon \rightarrow 0}\left\|\nabla \psi_{\varepsilon}(x)-\nabla \psi_{0}^{i}(x)-\left(\nabla_{y} \psi_{1}^{i}\right)\left(x, \frac{x}{\varepsilon}\right)\right\|_{L^{2}\left(\Omega_{\varepsilon}\right)^{n}}=0
$$

в соответствии с [9, доказательство теоремы 1.8$]$.

Следовательно, неравенство (2.8) можно переписать в виде

$$
\begin{gathered}
\int_{\Omega} \chi_{\varepsilon} a\left(\frac{x}{\varepsilon}, \nabla \psi_{0}^{i}+\left(\nabla_{y} \psi_{1}^{i}\right)^{\varepsilon}+\nabla v_{0}+\nabla_{y} v_{1}^{\varepsilon}\right)\left(\chi_{\varepsilon} \nabla v_{0}+\chi_{\varepsilon} \nabla_{y} v_{1}^{\varepsilon}-\chi_{\varepsilon} \nabla u_{\varepsilon}\right) d x+\sigma_{\varepsilon}^{i} \\
\geqslant \int_{\Omega} f\left(\chi_{\varepsilon} v_{0}-\chi_{\varepsilon} u_{\varepsilon}\right) d x+\sigma_{\varepsilon}
\end{gathered}
$$


где $\lim _{i \rightarrow \infty} \lim _{\varepsilon \rightarrow 0} \sigma_{\varepsilon}^{i}=0$ в силу (1.6) и (2.10). Используя определение (1.10), соотношения (2.5) и утверждение 1.1 , перейдем в неравенстве (2.11) к пределу при $\varepsilon \rightarrow 0$, поскольку из (1.6) следует, что

$$
\begin{aligned}
& \left|\chi a\left(y, \nabla \psi_{0}^{i}+\nabla_{y} \psi_{1}^{i}+\nabla v_{0}+\nabla_{y} v_{1}\right)\right| \\
& \quad \leqslant C\left|\chi\left(\nabla \psi_{0}^{i}+\nabla_{y} \psi_{1}^{i}+\nabla v_{0}+\nabla_{y} v_{1}\right)\right| \text { п.в. в } \Omega \times Y,
\end{aligned}
$$

и поэтому вектор-функцию $\chi a\left(y, \nabla \psi_{0}^{i}+\nabla_{y} \psi_{1}^{i}+\nabla v_{0}+\nabla_{y} v_{1}\right)$ (после непосредственной проверки, что эта вектор-функция непрерывна по $x \in \bar{\Omega}$ для п.в. $y \in Y$ ) можно рассматривать как тестовую вектор-функцию $\varphi(x, y)$ из определения (1.10). Следовательно, переходя в неравенстве (2.11) к пределу (по подпоследовательности из утверждения 1.1), имеем

$$
\begin{gathered}
\int_{\Omega} \int_{Y} \chi a\left(y, \nabla \psi_{0}^{i}+\nabla_{y} \psi_{1}^{i}+\nabla v_{0}+\nabla_{y} v_{1}\right)\left(\chi \nabla v_{0}+\chi \nabla_{y} v_{1}-\chi \nabla u_{0}-\chi \nabla_{y} u_{1}\right) d y d x \\
\quad+\lim _{\varepsilon \rightarrow 0} \sigma_{\varepsilon}^{i} \geqslant \int_{\Omega} \int_{Y} f\left(\chi v_{0}-\chi u_{0}\right) d y d x
\end{gathered}
$$

(для фиксированного $i$ ), где учтено также, что в определении (1.10) можно выбирать тестовую функцию $\varphi(=f(x))$ из $L^{2}(\Omega)$ (см. [8], [9]).

Переходя в последнем неравенстве к пределу по $i$ и используя (1.6) и (2.9), получаем, что $u_{0}$ и $u_{1}$ из утверждения 1.1 удовлетворяют неравенству (1.15) для гладких и финитных функций $v_{0}$ и $v_{1}, v_{0} \in K_{0}$. Пусть $\left(w_{0}, w_{1}\right) \in K_{2}$. В соответствии с (2.1) найдутся такие последовательности $\left\{v_{0}^{i}\right\} \subset C_{0}^{\infty}(\Omega) \cap K_{0}$ и $\left\{v_{1}^{i}\right\} \subset$ $C_{0}^{\infty}\left(\Omega ; C_{P}^{\infty}(Y) / \chi \mathbb{R}\right)$, что

$$
\lim _{i \rightarrow \infty}\left\|\nabla w_{0}+\nabla_{y} w_{1}-\nabla v_{0}^{i}-\nabla_{y} v_{1}^{i}\right\|_{L^{2}(\Omega \times F)^{n}}=0 .
$$

Таким образом, учитывая данное соотношение и (1.6), заключаем, что неравенство (1.15) выполнено и для каждого $\left(w_{0}, w_{1}\right) \in K_{2}$, поскольку это неравенство вьполнено для $v_{0}=v_{0}^{i}$ и $v_{1}=v_{1}^{i}$ при каждом $i$ и можно перейти к пределу по $i$, что завершает доказательство леммы.

ДОКАЗАТЕЛЬСТВО УТВЕРЖДЕНИЯ 1.2. В соответствИИ с Леммой 2.1 существует некоторое решение $\left(u_{0}, u_{1}\right) \in K_{2}$ вариационного неравенства (1.15) для всех $\left(v_{0}, v_{1}\right) \in K_{2}$. Фиксируем $\left(w_{0}, w_{1}\right) \in K_{2}$ и выберем

$$
\left(v_{0}, v_{1}\right)=\left(u_{0}+\sigma\left(w_{0}-u_{0}\right), u_{1}+\sigma\left(w_{1}-u_{1}\right)\right), \quad 0<\sigma \leqslant 1,
$$

в неравенстве из (1.15). В этом случае имеем $\left(v_{0}, v_{1}\right) \in K_{2}$ (поскольку множество $K_{0}$ выпукло) и неравенство из (1.15) принимает вид

$$
\begin{array}{r}
\int_{\Omega} \int_{F} a\left(y, \nabla \psi_{0}+\nabla_{y} \psi_{1}+\nabla u_{0}+\sigma \nabla\left(w_{0}-u_{0}\right)+\nabla_{y} u_{1}+\sigma \nabla_{y}\left(w_{1}-u_{1}\right)\right) \\
\times\left(\nabla w_{0}+\nabla_{y} w_{1}-\nabla u_{0}-\nabla_{y} u_{1}\right) d y d x \geqslant \int_{\Omega} \int_{F} f\left(w_{0}-u_{0}\right) d y d x,
\end{array}
$$

где $\left(w_{0}, w_{1}\right) \in K_{2}$. Используя (1.6), в этом неравенстве можно перейти к пределу при $\sigma \rightarrow 0$ и заключить, что $\left(u_{0}, u_{1}\right) \in K_{2}$ является решением вариационного неравенства (1.16). 
Пусть $\left(u_{0}, u_{1}\right) \in K_{2}-$ некоторое решение задачи (1.16). Из соотношения (1.6) следует, что $a(y, \xi)(\xi-\zeta) \geqslant a(y, \zeta)(\xi-\zeta)$ п.в. в $F$ для всех $\xi, \zeta \in \mathbb{R}^{n}$. Выберем в этом неравенстве $\xi=\nabla \psi_{0}+\nabla_{y} \psi_{1}+\nabla v_{0}+\nabla_{y} v_{1}$ и $\zeta=\nabla \psi_{0}+\nabla_{y} \psi_{1}+\nabla u_{0}+\nabla_{y} u_{1}$ для п.в. $x \in \Omega$ и п.в. $y \in F$ и проинтегрируем результат по $\Omega \times F$. В этом случае имеем

$$
\begin{aligned}
\int_{\Omega} \int_{F} a( & \left., \nabla \psi_{0}+\nabla_{y} \psi_{1}+\nabla v_{0}+\nabla_{y} v_{1}\right) \\
& \times\left(\nabla v_{0}+\nabla_{y} v_{1}-\nabla u_{0}-\nabla_{y} u_{1}\right) d y d x \\
\geqslant & \int_{\Omega} \int_{F} a\left(y, \nabla \psi_{0}+\nabla_{y} \psi_{1}+\nabla u_{0}+\nabla_{y} u_{1}\right) \\
& \times\left(\nabla v_{0}+\nabla_{y} v_{1}-\nabla u_{0}-\nabla_{y} u_{1}\right) d y d x \geqslant \int_{\Omega} \int_{F} f\left(v_{0}-u_{0}\right) d y d x
\end{aligned}
$$

для $\left(v_{0}, v_{1}\right) \in K_{2}$, поскольку $\left(u_{0}, u_{1}\right) \in K_{2}$ является решением задачи (1.16). Таким образом, вариационное неравенство (1.15) для всех $\left(v_{0}, v_{1}\right) \in K_{2}$ эквивалентно вариационному неравенству (1.16). Это утверждение является аналогом леммы Минти (см. [20, лемма III.1.5]) для двухмасштабного вариационного неравенства (1.16).

Предположим, что $\left(u_{0}^{1}, u_{1}^{1}\right) \in K_{2}$ и $\left(u_{0}^{2}, u_{1}^{2}\right) \in K_{2}$ являются решениями вариационного неравенства (1.16). Рассмотрим это неравенство для $\left(u_{0}^{1}, u_{1}^{1}\right)$ и выберем $\left(v_{0}, v_{1}\right)=\left(u_{0}^{2}, u_{1}^{2}\right)$. Аналогично, рассмотрим это неравенство для $\left(u_{0}^{2}, u_{1}^{2}\right)$ и выберем $\left(v_{0}, v_{1}\right)=\left(u_{0}^{1}, u_{1}^{1}\right)$. Суммируя эти неравенства, получаем

$$
\begin{aligned}
& \int_{\Omega} \int_{F}\left(a\left(y, \nabla \psi_{0}+\nabla_{y} \psi_{1}+\nabla u_{0}^{1}+\nabla_{y} u_{1}^{1}\right)\right. \\
& \left.\quad-a\left(y, \nabla \psi_{0}+\nabla_{y} \psi_{1}+\nabla u_{0}^{2}+\nabla_{y} u_{1}^{2}\right)\right)\left(\nabla u_{0}^{1}+\nabla_{y} u_{1}^{1}-\nabla u_{0}^{2}-\nabla_{y} u_{1}^{2}\right) d y d x \leqslant 0 .
\end{aligned}
$$

В силу условия строгой монотонности из (1.6) это неравенство является фактически равенством, которое подразумевает, что

$$
\nabla u_{0}^{1}+\nabla_{y} u_{1}^{1}=\nabla u_{0}^{2}+\nabla_{y} u_{1}^{2} \text { п.в. в } \Omega \times F .
$$

Следовательно, выполнено равенство $\left\|\left(u_{0}^{1}-u_{0}^{2}, u_{1}^{1}-u_{1}^{2}\right)\right\|_{2}=0$, и поэтому $u_{0}^{1}=u_{0}^{2}$ и $u_{1}^{1}=u_{1}^{2}$, поскольку равенство (1.13) определяет норму на $H_{2}$ (в соответствии c [9]), что доказывает единственность решения задачи (1.16). Утверждение доказано.

ДОКАЗАТЕЛЬСТВО УТВЕРЖДЕНИЯ 1.3. Известно [1, гл. $2, \S 8.1]$, что для фиксированного $\xi \in \mathbb{R}^{n}$ сушествует единственное решение вариационного неравенства (1.17) и это неравенство эквивалентно задаче (1.18).

Фиксируем $\xi \in \mathbb{R}^{n}$. Тогда для завершения доказательства утверждения достаточно проверить, что $\nabla_{y} U\left(\xi_{i}\right) \rightarrow \nabla_{y} U(\xi)$ слабо в $L_{P}^{2}(F)^{n}$ при $i \rightarrow \infty$, если $\left|\xi_{i}-\xi\right| \rightarrow 0$ при $i \rightarrow \infty$, где $U\left(\xi_{i}\right) \in H_{P}^{1}(F) / \mathbb{R}$ является единственным решением задачи (1.18) для $\xi_{i} \in \mathbb{R}^{n}$ и $U(\xi)$ - единственное решение задачи (1.18) для $\xi \in \mathbb{R}^{n}$. Из соотношений (1.6) и (1.18) для $V=U(\xi)$ следует, что

$$
\begin{aligned}
\alpha\left\|\nabla_{y} U(\xi)\right\|_{L_{P}^{2}(F)^{n}}^{2} & \leqslant \int_{F}\left(a\left(y, \nabla_{y} U\right)-a\left(y, \xi+\nabla_{y} U\right)\right) \nabla_{y} U d y \\
& \leqslant\left\|a\left(y, \nabla_{y} U\right)-a\left(y, \xi+\nabla_{y} U\right)\right\|_{L_{P}^{2}(F)^{n}}\left\|\nabla_{y} U(\xi)\right\|_{L_{P}^{2}(F)^{n}}
\end{aligned}
$$


и поэтому $\left\|\nabla_{y} U(\xi)\right\|_{L_{P}^{2}(F)^{n}} \leqslant(\beta \sqrt{\theta} / \alpha)|\xi|$ (см. (1.20)). Таким образом, можно считать, что последовательность $\left\{\nabla_{y} U\left(\xi_{i}\right)\right\} \subset L_{P}^{2}(F)^{n}$ является равномерно (по $i$ ) ограниченной и найдется такое $W \in H_{P}^{1}(F) / \mathbb{R}$, что (после выделения подпоследовательности) при $i \rightarrow \infty$

$$
\nabla_{y} U\left(\xi_{i}\right) \rightarrow \nabla_{y} W \quad \text { слабо в } L_{P}^{2}(F)^{n} .
$$

Рассмотрим задачу (1.18) для $\xi_{i} \in \mathbb{R}^{n}$. Известно [1], [20], что эта задача эквивалентна вариационному неравенству для $U\left(\xi_{i}\right) \in H_{P}^{1}(F) / \mathbb{R}:$

$$
\int_{F} a\left(y, \xi_{i}+\nabla_{y} V\right)\left(\nabla_{y} V-\nabla_{y} U\left(\xi_{i}\right)\right) d y \geqslant 0 \quad \forall V \in H_{P}^{1}(F) / \mathbb{R} .
$$

Для фиксированного $V \in H_{P}^{1}(F) / \mathbb{R}$ в этом неравенстве можно перейти к пределу по $i$ (используя только $(2.12)$, поскольку $a\left(y, \xi_{i}+\nabla_{y} V\right) \rightarrow a\left(y, \xi+\nabla_{y} V\right)$ сильно в $\left.L_{P}^{2}(F)^{n}\right)$ и заключить, что $W=U(\xi)$ в силу единственности решения задачи (1.18). Утверждение доказано.

ДОКАЗАТЕЛЬСТВО УТВЕРЖДЕНИЯ 1.4. Из сЛабой неПрерЬВности фУнКциИ $U(\xi)$ и сепарабельности пространства $H_{P}^{1}(F) / \mathbb{R}$ (поскольку $F$ является компактным многообразием) следует сильная измеримость этой функции по теореме Петтиса (см., например, [28]).

Из (1.6) следует, что $0 \leqslant a(y, \xi)(\xi-\zeta)-a(y, \zeta)(\xi-\zeta)$ п.в. в $F$ для каждого $\xi, \zeta \in \mathbb{R}^{n}$. Выберем в этом неравенстве $\xi=\nabla \psi_{0}+\nabla u_{0}+\nabla_{y} U\left(\nabla \psi_{0}+\nabla u_{0}\right)$ и $\zeta=\nabla \psi_{0}+\nabla u_{0}+\nabla_{y} \psi_{1}+\nabla_{y} u_{1}$ для п.в. $x \in \Omega$ и п.в. $y \in F$ и проинтегрируем результат по $\Omega \times F$. В этом случае имеем

$$
\begin{aligned}
0 \leqslant & \int_{\Omega} \int_{F} a\left(y, \eta+\nabla_{y} U(\eta)\right)\left(\nabla_{y} U(\eta)-\nabla_{y} \psi_{1}-\nabla_{y} u_{1}\right) d y d x \\
& -\int_{\Omega} \int_{F} a\left(y, \eta+\nabla_{y} \psi_{1}+\nabla_{y} u_{1}\right)\left(\nabla_{y} U(\eta)-\nabla_{y} \psi_{1}-\nabla_{y} u_{1}\right) d y d x
\end{aligned}
$$

где введено обозначение $\eta=\nabla \psi_{0}+\nabla u_{0}$.

Выбирая $v_{0}=u_{0}$ и $v_{1}=U\left(\nabla \psi_{0}+\nabla u_{0}\right)-\psi_{1}$ в неравенстве из $(1.16)$, получаем

$$
\int_{\Omega} \int_{F} a\left(y, \eta+\nabla_{y} \psi_{1}+\nabla_{y} u_{1}\right)\left(\nabla_{y} U(\eta)-\nabla_{y} \psi_{1}-\nabla_{y} u_{1}\right) d y d x \geqslant 0 .
$$

Выбирая $\xi=\nabla \psi_{0}+\nabla u_{0}$ и $V=U\left(\nabla \psi_{0}+\nabla u_{0}\right)-\psi_{1}-u_{1}$ (для п.в. $\left.x \in \Omega\right)$ в $(1.18)$ и интегрируя результат по $\Omega$, имеем равенство

$$
\int_{\Omega} \int_{F} a\left(y, \eta+\nabla_{y} U(\eta)\right)\left(\nabla_{y} U(\eta)-\nabla_{y} \psi_{1}-\nabla_{y} u_{1}\right) d y d x=0 .
$$

Используя это равенство и соотношение (2.14), заключаем, что в (2.13) выполнено равенство, и поэтому

$$
\nabla_{y} U\left(\nabla \psi_{0}+\nabla u_{0}\right)=\nabla_{y} \psi_{1}+\nabla_{y} u_{1} \text { п.в. в } \Omega \times F
$$

в силу условия строгой монотонности из (1.6), что с учетом (2.3) завершает доказательство утверждения. 
ДОКАЗАТЕЛЬСТВО ТЕОРЕМЫ 1.5. Используя утверждение 1.4 и выбирая $v_{1}=u_{1}$ в (1.16), заключаем, что $u_{0}$ (определенное в утверждении 1.1) является решением осредненной задачи (1.19), а $\hat{u}_{0}=\psi_{0}+u_{0}$ является решением осредненной задачи (1.21).

Из $(1.6)$ следует, что $0 \leqslant(a(y, \xi)-a(y, \zeta))(\xi-\zeta)$ п.в. в $F$ для каждого $\xi, \zeta \in \mathbb{R}^{n}$. Выберем в этом неравенстве $\xi=\eta+\nabla_{y} U(\eta)$ и $\zeta=\tau+\nabla_{y} U(\tau)$ для п.в. $y \in F$ и $\eta, \tau \in \mathbb{R}^{n}$ (где $U(\eta)$ и $U(\tau)$ - решения задачи (1.18)) и проинтегрируем результат по $F$. В этом случае имеем

$$
\begin{aligned}
0 \leqslant & \int_{F}\left(a\left(y, \eta+\nabla_{y} U(\eta)\right)-a\left(y, \tau+\nabla_{y} U(\tau)\right)\right) \\
& \times\left(\eta+\nabla_{y} U(\eta)-\tau-\nabla_{y} U(\tau)\right) d y .
\end{aligned}
$$

Учитывая (1.18) и определение $(1.20)$ в этом неравенстве, получаем для $\eta, \tau \in \mathbb{R}^{n}$ неравенство $0 \leqslant(A(\eta)-A(\tau))(\eta-\tau)$, что доказьвает монотонность вектор-функции $A(\eta)$.

Предположим, что в (2.15) выполнено равенство. Тогда из (1.6) имеем $\eta-\tau+$ $\nabla_{y} U(\eta)-\nabla_{y} U(\tau)=0$ п.в. в $F$. Используя 1 -периодичность, продолжим это равенство на $F_{1}$. Тогда $\nabla_{y}((\eta-\tau) y+U(\eta)-U(\tau))=0$, и поэтому $(\eta-\tau) y+U(\eta)-$ $U(\tau)=C$, поскольку $F_{1}$ связно. Функция $U(\eta)-U(\tau)$ определена с точностью до постоянной, и можно считать, что $C=0$. Таким образом, выполнено равенство $(\tau-\eta) y=U(\eta)-U(\tau)$, и поэтому $\eta=\tau$, поскольку функция $U(\eta)-U(\tau)$ является 1-периодической, что доказывает строгую монотонность вектор-функции $A(\eta)$.

Таким образом, решение $u_{0}$ осредненной задачи (1.19) (определенное в утверждении 1.1) является единственным. Тогда непосредственно из утверждений 1.1-1.4 следует, что

$$
\chi_{\varepsilon} \hat{u}_{\varepsilon} \stackrel{2}{\longrightarrow} \chi \hat{u}_{0}, \quad \chi_{\varepsilon} \nabla \hat{u}_{\varepsilon} \stackrel{2}{\longrightarrow} \chi \nabla \hat{u}_{0}+\chi \nabla_{y} U\left(\nabla \hat{u}_{0}\right)
$$

при $\varepsilon \rightarrow 0$ без выделения подпоследовательностей, поскольку предельные функции определены однозначно.

Кроме того, $\chi_{\varepsilon} \hat{u}_{\varepsilon} \rightarrow \theta \hat{u}_{0}$ слабо в $L^{2}(\Omega)$, поскольку в определении (1.10) можно выбрать тестовую функцию $\varphi$ из $L^{2}(\Omega)$ (см. [8], [9]). Тогда, используя лемму о компактности [3, лемма 3.3$]$, получаем

$$
\left\|\hat{u}_{\varepsilon}-\hat{u}_{0}\right\|_{L^{2}\left(\Omega_{\varepsilon}\right)} \rightarrow 0, \quad \varepsilon \rightarrow 0,
$$

что завершает доказательство теоремы.

Отметим, что доказано также и следующее утверждение.

УТВЕРЖДЕНИЕ 2.2. Пусть $\left(u_{0}, u_{1}\right) \in K_{2}$ является решением вариационного неравенства (1.16). Тогда $\left(u_{0}, u_{1}\right)=\left(u_{0}, U\left(\nabla \psi_{0}+\nabla u_{0}\right)-\psi_{1}\right)$, где $u_{0} \in K_{0}$ является единственным решением вариационного неравенства $(1.19), U(\xi)$ является единственным (для каждого $\xi \in \mathbb{R}^{n}$ ) решением задачи (1.18), а $\psi_{0}$ и $\psi_{1}$ определены в утверждении 1.1.

На самом деле верно и обратное утверждение.

УТВЕРЖДЕНИЕ 2.3. Пусть $u_{0} \in K_{0}$ является единственным решением вариационного неравенства (1.19), $U(\xi)$ является единственным (для каждого $\left.\xi \in \mathbb{R}^{n}\right)$ решением задачи (1.18), а $\psi_{0}$ и $\psi_{1}$ определены в утвержсдении 1.1 . Тогда $\left(u_{0}, u_{1}\right)=\left(u_{0}, U\left(\nabla \psi_{0}+\nabla u_{0}\right)-\psi_{1}\right)$ является единственным решением вариационного неравенства (1.18). 
ДоКАЗАТЕЛЬСтво. Напомним, что $\psi_{0}$ и $\psi_{1}$ определены однозначно в соответствии с предположениями. Пусть $\left(v_{0}, v_{1}\right) \in K_{2}$ и $u_{1}=U\left(\nabla \psi_{0}+\nabla u_{0}\right)-\psi_{1}$. Выберем $\xi=\nabla \psi_{0}+\nabla u_{0}$ и $V=v_{1}-u_{1}$ (для п.в. $x \in \Omega$ ) в задаче (1.18) и проинтегрируем результат по $\Omega$. Тогда, добавляя полученное соотношение к неравенству из (1.19), получим в точности вариационное неравенство (1.16), которое имеет единственное решение в соответствии с доказательством утверждения 1.2 , что завершает доказательство.

ДокАЗАТЕЛЬСТво ТЕОРЕмЫ 1.6. Доказательство этой теоремы повторяет доказательство теоремы 1.5 , в котором просто не учитывается неотрицательность рассматриваемых функций. Здесь полезно еще раз напомнить, что вариационное уравнение (1.22) эквивалентно вариационному неравенству (1.7), в котором $K$ заменено на $H_{D}^{1}\left(\Omega_{\varepsilon}\right)$.

ДОКАЗАТЕЛЬСТВО ТЕОРЕМЫ 1.7. Доказательство этой теоремы также повторяет доказательство теоремы 1.5. Следует только отметить, что $u_{\varepsilon}=\hat{u}_{\varepsilon}-\widehat{\psi}_{\varepsilon} \in$ $H_{D}^{1}\left(\Omega_{\varepsilon}\right)$ в соответствии с $(1.22)$, но $\hat{u}_{\varepsilon}$ может не принадлежать пространству $H_{D}^{1}\left(\Omega_{\varepsilon}\right)$, поскольку $\left\{\widehat{\psi}_{\varepsilon}\right\} \subset H^{1}\left(\Omega_{\varepsilon}\right)$. Тогда

$$
\left\|u_{\varepsilon}-u_{0}\right\|_{L^{2}\left(\Omega_{\varepsilon}\right)} \rightarrow 0, \quad \varepsilon \rightarrow 0
$$

как и в доказательстве теоремы 1.5 , а из результатов работы [3, лемма 2.3] следует, что для каждого $\omega \Subset \Omega$ выполнено соотношение

$$
\left\|\hat{u}_{\varepsilon}-\hat{u}_{0}\right\|_{L^{2}\left(\Omega_{\varepsilon} \cap \omega\right)} \rightarrow 0, \quad \varepsilon \rightarrow 0 .
$$

Разумеется, и $\left\|\hat{u}_{\varepsilon}-\hat{u}_{0}\right\|_{L^{2}\left(\Omega_{\varepsilon}\right)} \rightarrow 0$, если $\left\|\widehat{\psi}_{\varepsilon}-\widehat{\psi}_{0}\right\|_{L^{2}\left(\Omega_{\varepsilon}\right)} \rightarrow 0$ при $\varepsilon \rightarrow 0$. Однако последнее соотношение для $\widehat{\psi}_{\varepsilon}$ не следует из (1.24) и (1.25) и может не выполняться в обшем случае для $\left\{\widehat{\psi}_{\varepsilon}\right\} \subset H^{1}\left(\Omega_{\varepsilon}\right)$.

ДОКАЗАТЕЛЬСТВО УТВЕРЖДЕНИЯ 1.8. Из соОтношений (1.6) и (1.28) следует, что

$$
b(y, \xi)-b(y, \zeta) \geqslant a(y, \zeta)(\xi-\zeta)
$$

п.в. в $F$ для любых $\xi, \zeta \in \mathbb{R}^{n}$ (см. [13, утверждения I.5.4 и I.5.5]). Более того, из предположений (1.6) также следует, что

$$
0 \leqslant b(y, \xi)-b(y, \zeta)-a(y, \zeta)(\xi-\zeta) \leqslant \frac{\beta}{2}|\xi-\zeta|^{2}
$$

п.в. в $F$ для любых $\xi, \zeta \in \mathbb{R}^{n}$. Действительно, используя непрерьвность (по $\xi$ ) функций $b(y, \xi)$ и $a(y, \xi)$, имеем соотношения

$$
\begin{aligned}
b(y, \xi)-b(y, \zeta) & =\int_{0}^{1} \frac{d}{d t} b(y, \zeta+t(\xi-\zeta)) d t \\
& =\int_{0}^{1} \nabla_{\xi} b(y, \zeta+t(\xi-\zeta)) d t(\xi-\zeta) \\
& =a(y, \zeta)(\xi-\zeta)+\int_{0}^{1}(a(y, \zeta+t(\xi-\zeta))-a(y, \zeta)) d t(\xi-\zeta),
\end{aligned}
$$

которые доказывают второе неравенство из (2.17), а первое неравенство из (2.17) эквивалентно неравенству (2.16). 
Пусть $u_{0} \in K_{0}$ является решением осредненной задачи (1.19) и $v_{0} \in K_{0}$. Выберем в неравенстве $(2.16) \xi=\nabla \psi_{0}+\nabla v_{0}+\nabla_{y} U\left(\nabla \psi_{0}+\nabla v_{0}\right)$ и $\zeta=\nabla \psi_{0}+\nabla u_{0}+$ $\nabla_{y} U\left(\nabla \psi_{0}+\nabla u_{0}\right)$ для п.в. $x \in \Omega$ и п.в. $y \in F$ и проинтегрируем результат по $\Omega \times F$. В этом случае получаем

$$
\begin{aligned}
& \int_{\Omega} \int_{F} b\left(y, \nabla \psi_{0}+\nabla v_{0}+\nabla_{y} U\left(\nabla \psi_{0}+\nabla v_{0}\right)\right) d y d x \\
& \quad-\int_{\Omega} \int_{F} b\left(y, \nabla \psi_{0}+\nabla u_{0}+\nabla_{y} U\left(\nabla \psi_{0}+\nabla u_{0}\right)\right) d y d x \\
& \geqslant \int_{\Omega} \int_{F} a\left(y, \eta+\nabla_{y} U(\eta)\right)\left(\nabla \psi_{0}+\nabla v_{0}+\nabla_{y} U\left(\nabla \psi_{0}+\nabla v_{0}\right)\right. \\
&\left.-\eta-\nabla_{y} U(\eta)\right) d y d x \geqslant \int_{\Omega} \int_{F} f\left(v_{0}-u_{0}\right) d y d x
\end{aligned}
$$

поскольку $u_{0}$ удовлетворяет неравенству из (1.19), в котором учтено утверждение 1.4 и выбрано $v_{1}=U\left(y, \nabla \psi_{0}(x)+\nabla v_{0}(x)\right)-\psi_{1}(x, y)$. Здесь $\eta=\nabla \psi_{0}+\nabla u_{0}$. Из этих неравенств следует, что

$$
\begin{aligned}
\int_{\Omega} \int_{F} & \left(b\left(y, \nabla \psi_{0}+\nabla v_{0}+\nabla_{y} U\left(\nabla \psi_{0}+\nabla v_{0}\right)\right)-f v_{0}\right) d x d y \\
& \geqslant \int_{\Omega} \int_{F}\left(b\left(y, \nabla \psi_{0}+\nabla u_{0}+\nabla_{y} U\left(\nabla \psi_{0}+\nabla u_{0}\right)\right)-f u_{0}\right) d y d x
\end{aligned}
$$

для всех $v_{0} \in K_{0}$. По определению это означает, что $u_{0} \in K$ является решением осредненной задачи минимизации (1.31).

Предположим, что $u_{0} \in K_{0}$ является решением осредненной задачи минимизации (1.31), и поэтому выполнено (2.18). Для фиксированного $\xi \in \mathbb{R}^{n}$ рассмотрим задачу минимизации (1.30). В соответствии с определением решения $U(\xi) \in H_{P}^{1}(F) / \mathbb{R}$ этой задачи выполнено неравенство

$$
\int_{F} b\left(y, \xi+\nabla_{y} V\right) d y \geqslant \int_{F} b\left(y, \xi+\nabla_{y} U(\xi)\right) d y \quad \forall V \in H_{P}^{1}(F) / \mathbb{R} .
$$

Пусть $\left(v_{0}, v_{1}\right) \in K_{2}$. Выбирая $\xi=\nabla \psi_{0}+\nabla v_{0}$ и $V=v_{1}$ (для п.в. $x \in \Omega$ в в $(2.19)$ и интегрируя результат по $\Omega$, имеем

$$
\begin{array}{rl}
\int_{\Omega} \int_{F} & b\left(y, \nabla \psi_{0}+\nabla v_{0}+\nabla_{y} v_{1}\right) d y d x \\
& \geqslant \int_{\Omega} \int_{F} b\left(y, \nabla \psi_{0}+\nabla v_{0}+\nabla_{y} U\left(\nabla \psi_{0}+\nabla v_{0}\right)\right) d y d x
\end{array}
$$

Учитывая это неравенство, из (2.18) получаем соотношение

$$
\begin{array}{rl}
\int_{\Omega} \int_{F} & b\left(y, \nabla \psi_{0}+\nabla v_{0}+\nabla_{y} v_{1}\right) d y d x \\
& \quad-\int_{\Omega} \int_{F} b\left(y, \nabla \psi_{0}+\nabla u_{0}+\nabla_{y} U\left(\nabla \psi_{0}+\nabla u_{0}\right)\right) d y d x \\
\geqslant & \int_{\Omega} \int_{F} f\left(v_{0}-u_{0}\right) d y d x
\end{array}
$$


Выберем в этом соотношении $v_{0}=u_{0}+\sigma\left(w_{0}-u_{0}\right)$ и $v_{1}=U\left(\nabla \psi_{0}+\nabla u_{0}\right)$, где $w_{0} \in K_{0}$ и $0<\sigma \leqslant 1$. Тогда, учитывая неравенство (2.17), в котором выбрано $\xi=\nabla \psi_{0}+\nabla u_{0}+\sigma \nabla\left(w_{0}-u_{0}\right)+\nabla_{y} U\left(\nabla \psi_{0}+\nabla u_{0}\right)$ и $\zeta=\nabla \psi_{0}+\nabla u_{0}+$ $\nabla_{y} U\left(\nabla \psi_{0}+\nabla u_{0}\right)$, имеем

$$
\begin{gathered}
\sigma \int_{\Omega} \int_{F} a\left(y, \nabla \psi_{0}+\nabla u_{0}+\nabla_{y} U\left(\nabla \psi_{0}+\nabla u_{0}\right)\right)\left(\nabla w_{0}-\nabla u_{0}\right) d y d x \\
+\sigma^{2} \int_{\Omega} \int_{F}\left|\nabla\left(w_{0}-u_{0}\right)\right|^{2} d x \geqslant \sigma \int_{\Omega} \int_{F} f\left(w_{0}-u_{0}\right) d y d x
\end{gathered}
$$

для $w_{0} \in K_{0}$, поскольку $\xi-\zeta=\sigma \nabla\left(w_{0}-u_{0}\right)$ для выбранных $\xi$ и $\zeta$. Сокрашая на $\sigma$ и переходя к пределу при $\sigma \rightarrow 0$ в неравенстве $(2.21)$, заключаем, что $u_{0} \in K_{0}$ является решением осредненной задачи (1.19), где учтено, что $\theta>0$ в силу предположений. Утверждение доказано.

ДОКАЗАТЕЛЬСТвО УТВЕРЖДЕНИЯ 1.9. Доказательство этого утверждения фактически повторяет доказательство утверждения 1.8. Действительно, решение $\left(u_{0}, u_{1}\right) \in K_{2}$ вариационного неравенства (1.16) является решением двухмасштабной задачи минимизации (1.32) в силу неравенства (2.16) с соответствуюшими $\xi$ и $\zeta$. Обратное утверждение следует из (2.20), утверждения 1.4 и неравенства (2.17).

ДОКАЗАТЕЛЬСТвО УТвЕРЖДЕНИЯ 1.13. Как и в доказательстве утверждения 1.1, проверяется, что последовательности $\left\{u_{\varepsilon}\right\}$ и $\left\{\nabla u_{\varepsilon}\right\}$ решений задачи (1.37) и градиентов этих решений равномерно ограничены в $L^{2}\left(\Omega_{\varepsilon}\right)$ и $L^{2}\left(\Omega_{\varepsilon}\right)^{n}$ соответственно. Тогда утверждения о сходимости следуют из результатов работы $[9$, доказательство теоремы 2.9].

Кроме того, $\chi_{\varepsilon} u_{\varepsilon} \rightarrow \theta u_{0}$ слабо в $L^{2}(\Omega)$ при $\varepsilon \rightarrow 0$, поскольку в определении (1.10) можно выбрать тестовую функцию $\varphi$ из $L^{2}(\Omega)$. Следовательно, используя лемму о компактности [3, лемма 2.3$]$, получаем

$$
\int_{\Omega_{\varepsilon}} \varphi u_{\varepsilon} d x \rightarrow \theta \int_{\Omega} \varphi u_{0} d x, \quad \varepsilon \rightarrow 0
$$

для $\varphi \in C_{0}^{\infty}(\Omega) \cap K_{0}$ (см. (1.19)). Интеграл, стоящий слева в этом соотношении, является неотрицательным в силу предположений (см. (1.37)), и поэтому неотрищателен интеграл, стоящий справа, что доказывает неотрицательность $u_{0}$ (поскольку $\varphi \in C_{0}^{\infty}(\Omega) \cap K_{0}$ произвольно) и утверждение.

ДОКАЗАТЕЛЬСТВО ТЕОРЕМЫ 1.14. В неравенстве из (1.38) можно выбрать тестовую функцию $v=v_{0}(x)+\varepsilon v_{1}(x, x / \varepsilon)+\varepsilon \delta e_{\varepsilon}$ (см. (1.14)) и повторить доказательство леммы 2.1. Кроме того, выполнены утверждения, аналогичные утверждениям 1.2-1.4 (без существенных изменений в доказательствах). Таким образом, доказательство теоремы повторяет доказательство теоремы 1.5.

ЗАмечАниЕ 2.4. Несложно проверить, что доказанные утверждения остаются выполненными, если вместо задачи (1.5) рассмотреть более общее вариационное неравенство для $\hat{u}_{\varepsilon} \in K_{\varepsilon}$ :

$$
\int_{\Omega_{\varepsilon}} a\left(\frac{x}{\varepsilon}, \nabla \hat{u}_{\varepsilon}\right)\left(\nabla \hat{v}-\nabla \hat{u}_{\varepsilon}\right) d x \geqslant \int_{\Omega_{\varepsilon}} f_{\varepsilon}\left(\hat{v}-\hat{u}_{\varepsilon}\right) d x \quad \forall \hat{v} \in K_{\varepsilon},
$$


где последовательность $\left\{f_{\varepsilon}\right\} \subset L^{2}\left(\Omega_{\varepsilon}\right)$ является равномерно ограниченной, $\chi_{\varepsilon} f_{\varepsilon} \rightarrow \theta f$ слабо в $L^{2}(\Omega)$ при $\varepsilon \rightarrow 0$ (см. [3, лемма 3.3]) и $f$ определено однозначно (разумеется, что при неоднозначном определении $f$ в соответствующих утверждениях следует рассматривать подходящие подпоследовательности решений). Это замечание позволяет обобщить доказанные утверждения на случай, когда в задаче $(2.22)$ выбрано $f_{\varepsilon}=F\left(x, x / \varepsilon, \hat{u}_{\varepsilon}, \nabla \hat{u}_{\varepsilon}\right)$ при подходящих предположениях для функции $F$. Аналогичное замечание относится и к задачам $(1.22),(1.26)$ и $(1.36)$.

ЗАмечаниЕ 2.5. Доказанные утверждения остаются выполненными без сушественных изменений и в случае, когда в задаче $(1.5)$ множество $K_{\varepsilon}$ заменяется множеством $K_{\varepsilon}^{-} \equiv\left\{\hat{v} \in H_{D}^{1}\left(\Omega_{\varepsilon}\right): \hat{v} \leqslant \psi_{\varepsilon}\right.$ п.в. на $\left.S_{\varepsilon}\right\}$. Это замечание позволяет обобщить доказанные утверждения на вариационные неравенства с двухсторонними ограничениями на границе $S_{\varepsilon}$. Аналогичное замечание относится и к задаче $(1.36)$.

Проверим наконец, что равенство (1.11) выполнено, если $\psi_{\varepsilon}$ определено равенством (1.33), в котором $\psi_{1}(x, y) \in L^{2}\left(\Omega ; L_{P}^{\infty}(F)\right), \nabla_{y} \psi_{1}(x, y), \nabla_{x} \psi_{1}(x, y) \in$ $L^{2}\left(\Omega ; L_{P}^{\infty}(F)^{n}\right), \psi_{\varepsilon}^{0} \in H_{0}^{1}(\Omega)$ и $\psi_{\varepsilon}^{0} \rightarrow \psi_{0}$ сильно в $H_{0}^{1}(\Omega)$ при $\varepsilon \rightarrow 0$. Действительно, в этом случае

$$
\lim _{\varepsilon \rightarrow 0}\left\|\nabla\left(\psi_{\varepsilon}^{0}-\psi_{0}\right)\right\|_{L^{2}\left(\Omega_{\varepsilon}\right)^{n}}=0
$$

Кроме того, из (2.6) и неравенства треугольника имеем

$$
\lim _{\varepsilon \rightarrow 0}\left\|\nabla \psi_{\varepsilon}\right\|_{L^{2}\left(\Omega_{\varepsilon}\right)^{n}}=\lim _{\varepsilon \rightarrow 0}\left\|\nabla \psi_{0}+\nabla_{y} \psi_{1}^{\varepsilon}\right\|_{L^{2}\left(\Omega_{\varepsilon}\right)^{n}}
$$

и (1.11) следует из равенства (1.35), доказанного в [16], что завершает доказательство утверждений, сформулированных во введении.

\section{Список литературы}

1. Лионс Ж.-Л. Некоторые методы решения нелинейных краевых задач. М.: Мир, 1972.

2. Сьярле $\Phi$. Математическая теория упругости. М.: Мир, 1992.

3. Allaire G., Murat F. Homogenization of the Neuman problem with non-isolated holes. Paris: Universite Pierre et Marie Curie, 1992.

4. Hedberg L. I. Spectral synthesis in Sobolev spaces, and uniqueness of solutions of the Dirichlet problem // Acta Math. 1981. V. 147. P. 237-264.

5. Mitrea M., Taylor M. Boundary layer methods for Lipschitz domains in Riemannian manifolds // J. Funct. Anal. 1999. V. 163. № 2. P. 181-251.

6. Аәранович M. C. Спектральные задачи для сильно эллиптических систем второго порядка в областях с гладкой и негладкой границей // УМН. 2002. Т. 57. № 5. С. 3-78.

7. Ding Z. A proof of the trace theorem of Sobolev spaces on Lipschitz domains // Proc. Amer. Math. Soc. 1996. V. 124. № 2. P. 591-600.

8. Nguetseng G. A general convergence result for a functional related to the theory of homogenization // SIAM J. Math. Anal. 1989. V. 20. P. 608-623.

9. Allaire G. Homogenization and two-scale convergence // SIAM J. Math. Anal. 1992. V. 23. P. $1482-1518$.

10. Темам Р. Уравнения Навье-Стокса. М.: Мир, 1981.

11. Бахвалов Н. С., Панасенко Г. П. Осреднение процессов в периодических средах. М.: Наука, 1984.

12. Allaire G., Briane M. Multiscale convergence and reiterated homogenization // Proc. Royal Soc. Edinburgh. 1996. V. 126A. P. 297-342.

13. Экланд И., Темам Р. Выпуклый анализ и вариационные проблемы. М.: Мир, 1979.

14. Braides A., Defranceschi A. Homogenization of multiple integrals. N. Y.: Oxford University Press, 1998. 
15. Пастухова C.E. Усреднение смешанной задачи с условием Синьорини для эллиптического оператора в перфорированной области // Матем. сб. 2001. Т. 192. № 2. С. 87-102.

16. Амосов A.A. О слабой сходимости одного класса быстроосциллирующих функций // Матем. заметки. 1997. Т. 62. №1. С. 145-150.

17. Zhikov $V . V$. On the homogenization of nonlinear variational problems in perforated domains // Russian J. Math. Physics. 1994. V. 2. № 3. P. 393-408.

18. ЖКиков B. B., Рычаго M.E. Усреднение нелинейных эллиптических уравнений второго порядка в перфорированных областях // Изв. РАН. Сер. матем. 1997. Т. 61. № 1. C. $69-88$.

19. Сандраков Г. В. Осреднение вариационных неравенств для задач с регулярным препятствием // Докл. РАН. 2004. Т. 397. № 2. С. 170-173.

20. Киндерлерер Д., Стампаккья Г. Введение в вариационные неравенства и их приложения. М.: Мир, 1983.

21. Санчес-Паленсия Э. Неоднородные среды и теория колебаний. М.: Мир, 1984.

22. Bensoussan A., Lions J.-L., Papanicolau G. Asymptotic analysis for periodic structures. Amsterdam: North-Holland, 1978.

23. Lions J.-L., Sanchez-Palencia E. Écoulement d'un fluide viscoplastique de Bingham dans un milieu poreux // J. Math. Pures Appl. 1981. V. 60. P. 341-360.

24. Attouch H., Picard $C$. Variational inequalities with varying obstacles: the general form of the limit problem // J. Func. Anal. 1983. V. 50. P. 329-386.

25. Dal Maso G., Trebeschi P. Г-limit of periodic obstacles // Acta Appl. Math. 2001. V. 65. P. $207-215$.

26. Ладыженская $O . A .$, Уральцева $H . H$. Линейные и квазилинейные уравнения эллиптического типа. М.: Наука, 1973.

27. Рид М., Саймон Б. Методы современной математической физики. Т. 1. М.: Мир, 1977.

28. Иосида К. Функциональный анализ. М.: Мир, 1967.

Киевский национальный университет

им. Т. Г. Шевченко

Поступило в редакцию

17.05 .2004 